\title{
Characterizations of Certain Types of Type 2 Soft Graphs
}

\author{
Khizar Hayat $(\mathbb{D}),{ }^{1}$ Bing-Yuan Cao $\left(\mathbb{D},{ }^{1,2}\right.$ Muhammad Irfan Ali $(\mathbb{D})^{3}$ \\ Faruk Karaaslan $\mathbb{D}^{4}{ }^{4}$ and Zejian Qin ${ }^{1}$ \\ ${ }^{1}$ School of Mathematics and Information Sciences, Guangzhou University, 510000 Guangzhou, China \\ ${ }^{2}$ Guangzhou Vocational College of Science and Technology, Guangzhou, Guangdong 510550, China \\ ${ }^{3}$ Department of Mathematics, Islamabad Model College for Girls, F-6/2, Islamabad, Pakistan \\ ${ }^{4}$ Department of Mathematics, Faculty of Sciences, Çankırı Karatekin University, 18100 Çankırı, Turkey
}

Correspondence should be addressed to Bing-Yuan Cao; caobingy@163.com

Received 4 March 2018; Accepted 29 August 2018; Published 20 September 2018

Academic Editor: Allan C. Peterson

Copyright (C) 2018 Khizar Hayat et al. This is an open access article distributed under the Creative Commons Attribution License, which permits unrestricted use, distribution, and reproduction in any medium, provided the original work is properly cited.

\begin{abstract}
The vertex-neighbors correspondence has an essential role in the structure of a graph. The type 2 soft set is also based on the correspondence of initial parameters and underlying parameters. Recently, type 2 soft graphs have been introduced. Structurally, it is a very efficient model of uncertainty to deal with graph neighbors and applicable in applied intelligence, computational analysis, and decision-making. The present paper characterizes type 2 soft graphs on underlying subgraphs (regular subgraphs, irregular subgraphs, cycles, and trees) of a simple graph. We present regular type 2 soft graphs, irregular type 2 soft graphs, and type 2 soft trees. Moreover, we introduce type 2 soft cycles, type 2 soft cut-nodes, and type 2 soft bridges. Finally, we present some operations on type 2 soft trees by presenting several examples to demonstrate these new concepts.
\end{abstract}

\section{Preliminaries and Introduction}

A graph $\mathscr{G}=(X, \mathscr{E})$ consists of a nonempty set of objects $X$, called vertices, and a set $\mathscr{E}$ of two element subsets of $X$ called edges. Two vertices $x$ and $y$ are adjacent if $\{x, y\} \in \mathscr{E}$. A loop is an edge that connects a vertex to itself. A simple graph is an unweighted, undirected graph containing no multiple edges or graph loops. A graph $\mathscr{G}^{\prime}=\left(X^{\prime}, \mathscr{E}^{\prime}\right)$ is said to be a subgraph of $\mathscr{G}=(X, \mathscr{E})$ if $X^{\prime} \subseteq X$ and $\mathscr{E}^{\prime} \subseteq \mathscr{E}$. The graph neighborhoods of a vertex $x$ in a graph is the set of all the vertices adjacent to $x$ including $x$ itself. The graph neighbors of a vertex $x$ in a graph are the set of all the vertices adjacent to $x$ excluding $x$ itself. The eccentricity of the vertex $x$ is the maximum distance from $x$ to any vertex. The distance between two vertices $x$ and $y$ in a graph is the number of edges in a shortest path, denoted by $d(x, y)$. The radius of a graph is the minimum eccentricity of any vertex $x$. A graph $\mathscr{G}=(X, \mathscr{E})$ is called a tree if it is connected and contains no cycles. Equivalently (and sometimes more useful), a tree is a connected graph on $m$ vertices with exactly $m-1$ edges. A forest is a disjoint union of trees. The degree of a vertex of a simple graph is the number of edges incident to the vertex.
A regular graph is a graph where each vertex has the same number of neighbors. A graph that is not a regular graph is called irregular graph. A graph is called neighborly irregular graph if no two adjacent vertices have the same degree. A complete graph is a graph in which each pair of graph vertices is connected by an edge. For basic definitions of graphs see [1-3].

Soft set theory [4], firstly initiated by Molodtsov, is a new mathematical tool for dealing with uncertainties. Some fruitful operations, soft set theory, are presented by Maji et al. [5] and Ali et al. [6]. We refer to Molodtsov's soft sets as type 1 soft sets (briefly T1SS). Let $A$ be a set of parameters that can have an arbitrary nature (numbers, functions, sets of words, etc.). Let $U$ be a universe and the power set of $U$ is denoted by $P(U)$. The soft set is defined as follows.

Definition 1 (see [5]). A pair $(S, A)$ is called a soft set over $U$, where $S$ is a mapping given by $S: A \longrightarrow P(U)$.

Note that the set of all T1SS over $U$ will be denoted by $\sigma(U)$. Many researchers take attention at applicability of soft sets in real and practical problems. In recent years, research 
on soft set theory has been rapidly developed, and great progress has been achieved, including works of soft sets in graph theory. Ali et al. [7] introduced a representation of graphs based on neighborhoods. Akram et al. introduced the concepts of soft graphs $[8,9]$ and soft trees [10]. Let $\mathscr{G}=$ $(X, \mathscr{E})$ be a simple graph, $A$ be any nonempty set.

Let $R \subseteq A \times X$ be an arbitrary relation from $A$ to $X$. A mapping $I$ from $A$ to $P(X)$ denoted as $I: A \longrightarrow P(X)$ and defined as $I(x)=\{y \in X \mid x R y\}$ and a mapping $J$ from $A$ to $P(\mathscr{E})$ denoted as $J: A \longrightarrow P(\mathscr{E})$ and defined as $J(x)=\{u v \in$ $\mathscr{E} \mid\{u, v\} \subseteq I(x)\}$. Then $(I, A)$ is a T1SS over $X$ and $(J, A)$ is a T1SS over $\mathscr{E}$. The notion of a soft graph is defined as follows.

Definition 2 (see [9]). A 4-tuple $G^{2}=(\mathscr{G}, I, J, A)$ is called a soft graph if it satisfies;

(i) $\mathscr{G}=(X, \mathscr{E})$ is a simple graph.

(ii) $A$ is a non-empty set of parameters.

(iii) $[I, A]$ is a T1SS over $X$.

(iv) $[J, A]$ is a $T 1 S S$ over $\mathscr{E}$.

(v) $(I(\alpha), J(\alpha))$ for all $\alpha \in A$, represents a subgraph of $\mathscr{G}$.

The soft graph $G^{2}=(\mathscr{G}, I, J, A)$ can also be written as $G^{2}=$ $(\mathscr{G}, I, J)=\{T(\alpha) \mid \alpha \in A\}$, where $T(\alpha)=(I(\alpha), J(\alpha)) \forall \alpha \in$ A.

Definition 3 (see $[9,10]$ ). Let $G^{2}$ be a soft graph of $\mathscr{G}$. Then $G^{2}$ is said to be a soft tree (resp., regular soft graph, irregular soft graph, neighborly irregular soft graph, soft cycle) if every $T(\alpha)$ is a tree (resp., regular graph, irregular graph, neighborly irregular graph, and cycle) for all $\alpha \in A$.

A soft graph $G^{2}$ is called a regular soft graph of degree $r$ if $T(\alpha)$ is a regular graph of degree $r$ for all $\alpha \in A$. In the rest of paper soft tree and soft cycle will be written as type 1 soft tree (briefly, T1ST) and type 1 soft cycle (briefly, T1SC), respectively. Some operations of T1STs are defined as follows.

Definition 4 (see [10]). Let $G^{2}=\left\langle I_{1}, J_{1}, A_{1}\right\rangle$ and $G^{\prime 2}=$ $\left\langle I_{2}, J_{2}, A_{2}\right\rangle$ be two T1STs of $\mathscr{G}$. Then $G^{\prime 2}$ is a type 1 soft subtree of $G^{2}$ if

(i) $A_{2} \subseteq A_{1}$;

(ii) for each $x \in A_{2}, T_{2}(x)$ is a subtree of $T_{1}(x)$.

Definition 5 (see [10]). Let $G_{1}^{2}=\left\langle I_{1}, J_{1}, A_{1}\right\rangle$ and $G_{2}^{2}=$ $\left\langle I_{2}, J_{2}, A_{2}\right\rangle$ be two T1STs of $\mathscr{G}$. The extended union of $G_{1}^{2}$ and $G_{2}^{2}$, denoted by $G_{1}^{2} \sqcup_{\varepsilon} G_{2}^{2}=G^{2}=\langle I, J, B\rangle$, where $B=A_{1} \cup A_{2}$, is defined $\forall \alpha \in B$, as

$$
\begin{aligned}
& I(\alpha)= \begin{cases}I_{1}(\alpha), & \text { if } \alpha \in A_{1}-A_{2} \\
I_{2}(\alpha), & \text { if } \alpha \in A_{2}-A_{1} \\
I_{1}(\alpha) \cup I_{2}(\alpha), & \text { if } \alpha \in A_{1} \cap A_{2}\end{cases} \\
& J(\alpha)= \begin{cases}J_{1}(\alpha), & \text { if } \alpha \in A_{1}-A_{2} \\
J_{2}(\alpha), & \text { if } \alpha \in A_{2}-A_{1} \\
J_{1}(\alpha) \cup J_{2}(\alpha), & \text { if } \alpha \in A_{1} \cap A_{2}\end{cases}
\end{aligned}
$$
$B\}$.

It can be written as $G_{1}^{2} \sqcup_{\varepsilon} G_{2}^{2}=\{T(\alpha)=(I(\alpha), J(\alpha)) \mid \alpha \epsilon$

Definition 6 (see [10]). Let $G_{1}^{2}=\left\langle I_{1}, J_{1}, A_{1}\right\rangle$ and $G_{2}^{2}=$ $\left\langle I_{2}, J_{2}, A_{2}\right\rangle$ be two T1STs in $\mathscr{G}$. The restricted intersection of $G_{1}^{2}=\left\langle I_{1}, J_{1}, A_{1}\right\rangle$ and $G_{2}^{2}=\left\langle I_{2}, J_{2}, A_{2}\right\rangle$, denoted by $G_{1}^{2} \Pi_{r} G_{2}^{2}=$ $G^{2}=\langle I, J, C\rangle$, where $B=A_{1} \cap A_{1}$, is defined $\forall \alpha \in B$, as $I(\alpha)=I_{1}(\alpha) \cap I_{2}(\alpha), J(\alpha)=J_{1}(\alpha) \cap J_{2}(\alpha), \forall \alpha \in A_{1} \cap A_{2}$. $B\}$.

It can be written as $G_{1}^{2} \Pi_{r} G_{2}^{2}=\{T(\alpha)=(I(\alpha), J(\alpha)) \mid \alpha \epsilon$

Definition 7 (see [10]). Let $G_{1}^{2}=\left\langle I_{1}, J_{1}, A_{1}\right\rangle$ and $G_{2}^{2}=$ $\left\langle I_{2}, J_{2}, A_{2}\right\rangle$ be two T1STs in $\mathscr{G}$. The AND operation of $G_{1}^{2}$ and $G_{2}^{2}$, denoted by $G_{1}^{2} \wedge G_{2}^{2}=G^{2}=\langle I, J, C\rangle$, where $C=A_{1} \times A_{2}$, is defined $\forall(\alpha, \beta) \in A_{1} \times A_{2}$, as $I(\alpha, \beta)=I_{1}(\alpha) \cap I_{2}(\beta)$, $J(\alpha, \beta)=J_{1}(\alpha) \cap J_{2}(\beta), \forall(\alpha, \beta) \in A_{1} \times A_{2}$.

Definition 8 (see [10]). Let $G_{1}^{2}=\left\langle I_{1}, J_{1}, A_{1}\right\rangle$ and $G_{2}^{2}=$ $\left\langle I_{2}, J_{2}, A_{2}\right\rangle$ be two T1STs in $\mathscr{G}$. The OR operation of $G_{1}^{2}$ and $G_{2}^{2}$, denoted by $G_{1}^{2} \vee G_{2}^{2}=G^{2}=\langle I, J, C\rangle$, where $C=A_{1} \times A_{2}$, is defined $\forall(\alpha, \beta) \in A_{1} \times A_{2}$, as $I(\alpha, \beta)=I_{1}(\alpha) \cup I_{2}(\beta)$, $J(\alpha, \beta)=J_{1}(\alpha) \cup J_{2}(\beta), \forall(\alpha, \beta) \in A_{1} \times A_{2}$.

Definition 9 (see [8]). Let $G^{2}=\langle I, J, A\rangle$ be a soft graph of $\mathscr{G}$. Then the complement of $G^{2}$ is denoted by $G^{2 c}$ and defined by $G^{2 c}=\left\langle I^{c}, J^{c}, A\right\rangle$, where $I^{c}(\alpha)=I(\alpha)$ and $J^{c}(\alpha)$ contain those edges which are not included in $J(\alpha)$.

A generalization of soft sets called type 2 soft sets is introduced by Chatterjee et al. [11]. Some results on type 2 soft sets are validated by Yang and Wang [12] and some new operation on typ-2 soft sets are defined by Khizar et al. [13]. Let $X$ be universe set and $E$ be the set of parameters. The definition of type 2 soft set is as follows:

Definition 10 (see [11]). Let $(U, E)$ be a soft universe and $\sigma(U)$ be the collection of all T1SS over $(U, E)$. Then a mapping $: A \longrightarrow \sigma(U), A \subseteq E$ is called a type 2 soft set (briefly $T 2 S S)$ over $(U, E)$ and it is denoted by $\left[S^{*}, A\right]$. In this case, corresponding to each parameter $\alpha \in A, S^{*}(\alpha)$ is a T1SS. Thus, for each $\alpha \in A$, there exists a T1SS, $\left(S_{\alpha}, L_{\alpha}\right)$ such that $S^{*}(\alpha)=\left(S_{\alpha}, L_{\alpha}\right)$ where $S_{\alpha}: L_{\alpha} \longrightarrow P(U)$ and $L_{\alpha} \subset E$. In this case, we refer to the parameter set $A$ as the "primary set of parameters", while the set of parameters $\cup L_{\alpha}$ is known as the "underlying set of parameters".

Let $\mathscr{G}=(X, \mathscr{E})$ be a simple graph. The set of all T1SS over $X$ is denoted by $\Gamma(X)$ and the set of all T1SS over $\mathscr{E}$ is denoted by $\Gamma(\mathscr{E})$. The set of neighbors of an element $x \in X$ is denoted by $\mathcal{N} \mathscr{B}_{x}$ and defined by $\mathscr{N} \mathscr{B}_{x}=\{z \in X \mid x z \in \mathscr{E}\}$. Then $\mathscr{N} \mathscr{B}_{A}=\bigcup_{x \in A} \mathscr{N} \mathscr{B}_{x}$, where $A \subset X$. Let a subset $\mathscr{R}$ of $\mathscr{N} \mathscr{B}_{A} \times X$ be an arbitrary relation from $\mathscr{N} \mathscr{B}_{A}$ to $X$. Let $\mathscr{G}=(X, \mathscr{E})$ be a simple graph. Khizar et al. [14] introduced type 2 soft graphs by using T2SSs over $X$ and T2SSs over $\mathscr{E}$.

Definition 11 (see [14]). Let $\mathscr{G}=(X, \mathscr{E})$ be a simple graph, $A \subset X$ and $\Gamma(X)$ be the collection of all T1SS over $X$. Then a mapping $I^{*}: A \longrightarrow \Gamma(X)$ is called a T2SS over $X$ and it is denoted by $\left[I^{*}, A\right]$. In this case, corresponding to each vertex $x \in A, I^{*}(x)$ is a T1SS. Thus, for each $x \in A$, there exists 
a T1SS, $\left(I_{x}, \mathscr{N} \mathscr{B}_{x}\right)$ such that $I^{*}(x)=\left(I_{x}, \mathcal{N} \mathscr{B}_{x}\right)$ where $I_{x}$ : $\mathcal{N} \mathscr{B}_{x} \longrightarrow P(X)$ can be defined as $I_{x}(z)=\{y \in X \mid z \mathscr{R} y\}$, for all $z \in \mathcal{N} \mathscr{B}_{x} \subseteq X$ and $\mathcal{N} \mathscr{B}_{x}$ is the set of all neighbors of $x \in A$. This T2SS is also called a vertex-neighbors induced type 2 soft set (briefly, $V N$-type 2 soft set) over $X$.

Definition 12 (see [14]). Let $\mathscr{G}=(X, \mathscr{E})$ be a simple graph, $A \subset X$ and $\Gamma(\mathscr{E})$ be the collection of all T1SS over $\mathscr{E}$. Let $\left[I^{*}, A\right]$ be a $V N$-type 2 soft set over $X$. Then a mapping $J^{*}: A \longrightarrow \Gamma(\mathscr{E})$ is called a T2SS over $\mathscr{E}$ and it is denoted by $\left[J^{*}, A\right]$. In this case, corresponding to each vertex $x \in$ $A, J^{*}(x)$ is a T1SS. Thus, for each $x \in A$, there exists a T1SS, $\left(J_{x}, \mathscr{N} \mathscr{B}_{x}\right)$ such that $J^{*}(x)=\left(J_{x}, \mathscr{N} \mathscr{B}_{x}\right)$ where $J_{x}$ : $\mathcal{N} \mathscr{B}_{x} \longrightarrow P(\mathscr{E})$ can be define as $J_{x}(z)=\{\alpha \beta \in \mathscr{E} \mid\{\alpha, \beta\} \subseteq$ $\left.I_{x}(z)\right\}$, for all $z \in \mathscr{N} \mathscr{B}_{x} \subseteq X$ and $\mathscr{N} \mathscr{B}_{x}$ is the set of all neighbors of $x \in A$. This T2SS is also called a $V N$-type 2 soft set over $\mathscr{E}$.

Definition 13 (see [14]). A 5-tuple $\mathscr{G}^{*}=\left(\mathscr{G}, I^{*}, J^{*}, A, \mathscr{N}_{\mathscr{B}}\right)$ is called a type 2 soft graph (briefly, T2SG) if it satisfies following conditions:

(i) $\mathscr{G}=(X, \mathscr{E})$ is a simple graph.

(ii) $A$ is a non-empty set of parameters.

(iii) $\left[I^{*}, A\right]$ is a $V N$-type 2 soft set over $X$.

(iv) $\left[J^{*}, A\right]$ is a $V N$-type 2 soft set over $\mathscr{E}$.

(v) T1SS corresponding to $\left(I^{*}(x), J^{*}(x)\right) \forall x \in A$, represents a type 1 soft graph.

A T2SG can also be represented by $\mathscr{G}^{*}=\left\langle I^{*}, J^{*}, A\right\rangle=$ $\left\{T^{*}(x) \mid x \in A\right\}$, where $T^{*}(x)=\left(T_{x}, \mathcal{N} \mathscr{B}_{x}\right)$ such that $T_{x}(z)=\left(I_{x}(z), J_{x}(z)\right), \forall z \in \mathscr{N} \mathscr{B}_{x}$.

\section{Certain Types of Type 2 Soft Graphs}

In this section, we present regular type 2 soft graphs, irregular type 2 soft graphs, and type 2 soft trees. Moreover, we introduce type 2 soft cycles, type 2 soft cut-nodes, and type 2 soft bridges.

Definition 14. Let $\mathscr{G}^{*}=\left(\mathscr{G}, I^{*}, J^{*}, A, \mathcal{N} \mathscr{B}_{A}\right)$ be a $T 2 S G$ of $\mathscr{G}$. Then $\mathscr{G}^{*}$ is said to be a regular T2SG if T1SG corresponding to every $T^{*}(\gamma)$ is a regular T1SG for all $\gamma \in A$. A T2SG $\mathscr{G}^{*}$ is called a regular T2SG of degree $r$ if T1SG corresponding to $T^{*}(\gamma)$ is a regular T1SG of degree $r$ for all $\gamma \in A$.

Example 15. Consider a graph $\mathscr{G}=(X, \mathscr{E})$ as shown in Figure 1. Let $A=\left\{e_{9}, e_{10}\right\}$. It may be written that $\mathcal{N} \mathscr{B}_{e_{9}}=\left\{e_{11}, e_{8}\right\}$ and $\mathscr{N}_{e_{10}}=\left\{e_{8}, e_{11}\right\}$. Let $\left[I^{*}, A\right]$ and $\left[J^{*}, A\right]$ be two T2SSs over $X$ and $\mathscr{E}$ respectively, such that $I^{*}(x)=\left(I_{x}, \mathcal{N} \mathscr{B}_{x}\right)$ and $J^{*}(x)=\left(J_{x}, \mathscr{N}_{x}\right)$ for all $x \in A$. Define $I_{e_{0}}(z)=\{y \in X \mid z \mathscr{R} y \Longleftrightarrow 5 \geq d(z, y) \geq 3\}$, $J_{e_{9}}(z)=\left\{\alpha \beta \in \mathscr{E} \mid\{\alpha, \beta\} \subseteq I_{e_{9}}(z)\right\}$ for all $z \in \mathscr{N}_{e_{e_{9}}} \subseteq X$, and $I_{e_{10}}(z)=\{y \in X \mid z \mathscr{R} y \Longleftrightarrow 5 \geq d(z, y) \geq 3\}$, $J_{e_{10}}(z)=\left\{\alpha \beta \in \mathscr{E} \mid\{\alpha, \beta\} \subseteq I_{e_{10}}(z)\right\}$ for all $z \in \mathscr{N}_{e_{e_{10}}} \subseteq X$. Then T2SSs $\left[I^{*}, A\right]$ and $\left[J^{*}, A\right]$ are as in the following $I^{*}\left(e_{9}\right)=\left\{\left\{e_{11},\left\{e_{4}, e_{5}, e_{6}, e_{7}\right\}\right\},\left\{e_{8},\left\{e_{1}, e_{3}, e_{4}, e_{2}\right\}\right\}\right\}, J^{*}\left(e_{9}\right)=$ $\left\{\left\{e_{11},\left\{e_{4} e_{5}, e_{5} e_{7}, e_{4} e_{6}, e_{7} e_{6}\right\}\right\},\left\{e_{8},\left\{e_{1} e_{2}, e_{3} e_{2}, e_{3} e_{4}, e_{1} e_{4}, e_{2} e_{4}\right.\right.\right.$, $\left.\left.\left.e_{1} e_{3}\right\}\right\}\right\}, I^{*}\left(e_{10}\right)=\left\{\left\{e_{11},\left\{e_{4}, e_{5}, e_{6}, e_{7}\right\}\right\},\left\{e_{8},\left\{e_{1}, e_{3}, e_{4}, e_{2}\right\}\right\}\right\}$,

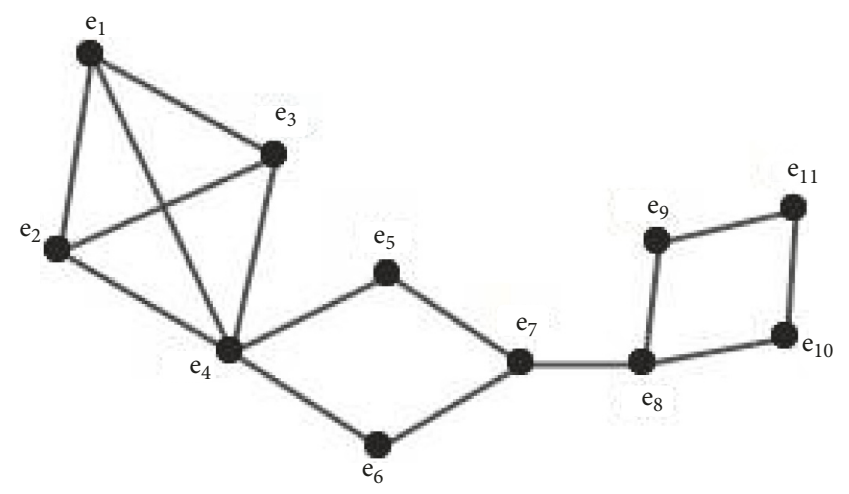

FIGURE 1: Simple graph.

$J^{*}\left(e_{10}\right)=\left\{\left\{e_{11},\left\{e_{4} e_{5}, e_{5} e_{7}, e_{4} e_{6}, e_{7} e_{6}\right\}\right\},\left\{e_{8},\left\{e_{1} e_{2}, e_{3} e_{2}, e_{3} e_{4}\right.\right.\right.$, $\left.\left.\left.e_{1} e_{4}, e_{2} e_{4}, e_{1} e_{3}\right\}\right\}\right\}$. One can check that $\mathscr{G}^{*}=\left(T^{*}\left(e_{9}\right), T^{*}\left(e_{10}\right)\right)$ is a regular T2SG of $\mathscr{G}$ as shown in Figure 2.

Theorem 16. Let $\mathscr{G}$ be a complete graph. Then every T2SG of $\mathscr{G}$ is a regular T2SG of $\mathscr{G}$.

Proof. Suppose $\mathscr{G}$ is a complete graph and $\mathscr{G}^{*}$ be a T2SG of $\mathscr{G}$. Let $\left(T_{\alpha}, \mathcal{N} \mathscr{B}_{\alpha}\right)$ be a $T 1 S G$ corresponding to $T^{*}(\alpha)$ for all $\alpha \in A$. Then $T_{\alpha}(x) \forall x \in \mathcal{N} \mathscr{B}_{\alpha}$ is a subgraph of $\mathscr{G}$. Since every subgraph of a complete graph is complete and every complete graph is regular. Therefore, $T_{\alpha}(x) \forall x \in \mathscr{N} \mathscr{B}_{\alpha}$ is a regular subgraph. This implies that TISG corresponding to $T^{*}(\alpha)$ for all $\alpha \in A$ is regular T1SG. Hence $\mathscr{G}^{*}$ is a regular T2SG of $\mathscr{G}$.

Note that if $\mathscr{G}^{*}$ is a regular T2SG of $\mathscr{G}$ then $\mathscr{G}$ need not be a complete graph.

Example 17. In Example 15, $\mathscr{G}^{*}$ is regular T2SG but $\mathscr{G}$ is not a complete graph.

Definition 18. Let $\mathscr{G}^{*}=\left(\mathscr{G}, I^{*}, J^{*}, A, \mathcal{N} \mathscr{B}_{A}\right)$ be a $T 2 S G$ of $\mathscr{G}$. Then the complement of $\mathscr{G}^{*}$ is denoted by $\mathscr{G}^{* c}$ and defined by $\mathscr{G}^{* c}=\left(T^{* c}\left(z_{1}\right), T^{* c}\left(z_{2}\right), . ., T^{* c}\left(z_{n}\right)\right)$ for all $x_{1}, x_{2}, . ., x_{n} \in A$, where T1SG corresponding to $T^{* c}\left(z_{i}\right)=\left(I^{* c}\left(z_{i}\right), J^{* c}\left(z_{i}\right)\right)$ is the complement of T1SG corresponding to $T^{*}\left(z_{i}\right)=$ $\left(I^{*}\left(z_{i}\right), J^{*}\left(z_{i}\right)\right)$ for all $z_{i} \in A(i=1$ to $n)$.

Example 19. Consider a graph $\mathscr{G}=(X, \mathscr{E})$ as shown in Figure 3. Let $A=\left\{e_{2}, e_{5}\right\}$. It may be written that $\mathcal{N} \mathscr{B}_{e_{2}}=$ $\left\{e_{1}, e_{3}, e_{4}\right\}$ and $\mathscr{N} \mathscr{B}_{e_{5}}=\left\{e_{4}, e_{6}\right\}$. Let $\left[I^{*}, A\right]$ and $\left[J^{*}, A\right]$ be two T2SSs over $X$ and $\mathscr{E}$ respectively, such that $I^{*}(x)=$ $\left(I_{x}, \mathscr{N}_{x}\right)$ and $J^{*}(x)=\left(J_{x}, \mathscr{N}_{x}\right)$ for all $x \in A$. Define $I_{e_{2}}(z)=\{y \in X \mid z \mathscr{R} y \Longleftrightarrow d(z, y) \leq 1\}, J_{e_{2}}(z)=\{\alpha \beta \in \mathscr{E} \mid$ $\left.\{\alpha, \beta\} \subseteq I_{e_{2}}(z)\right\}$ for all $z \in \mathscr{N}_{e_{2}} \subseteq X$, and $I_{e_{5}}(z)=\{y \in X \mid$ $z \mathscr{R} y \Longleftrightarrow d(z, y)=2\}, J_{e_{5}}(z)=\left\{\alpha \beta \in \mathscr{E} \mid\{\alpha, \beta\} \subseteq I_{e_{5}}(z)\right\}$ for all $z \in \mathscr{N}_{e_{5}} \subseteq X$. Then T2SSs $\left[I^{*}, A\right]$ and $\left[J^{*}, A\right]$ are as follows:

$$
\begin{aligned}
& I^{*}\left(e_{2}\right)=\left\{\left\{e_{1},\left\{e_{1}, e_{3}, e_{6}, e_{2}\right\}\right\},\left\{e_{3},\left\{e_{1}, e_{3}, e_{7}, e_{2}\right\}\right\},\right. \\
& \left.\quad\left\{e_{4},\left\{e_{7}, e_{5}, e_{4}, e_{2}\right\}\right\}\right\},
\end{aligned}
$$




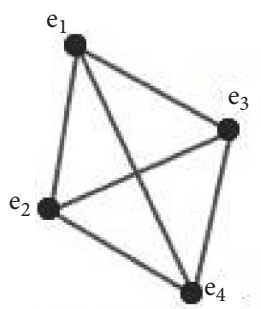

$\mathrm{T}_{\mathrm{e} 9}\left(\mathrm{e}_{8}\right)$

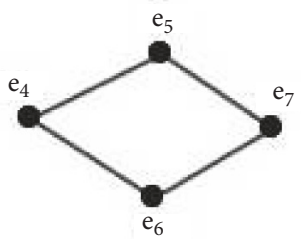

$\mathrm{T}_{\mathrm{e} 9}\left(\mathrm{e}_{11}\right)$

$\mathrm{T}^{*}\left(\mathrm{e}_{9}\right)$

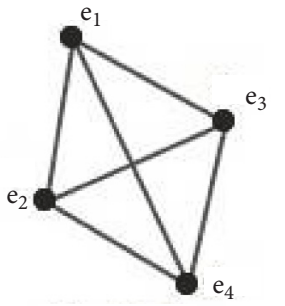

$\mathrm{T}_{\mathrm{e} 10}\left(\mathrm{e}_{8}\right)$

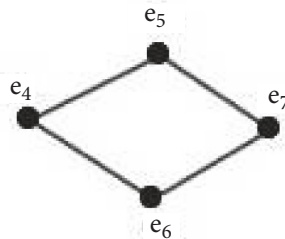

$\mathrm{T}_{\mathrm{e} 10}\left(\mathrm{e}_{11}\right)$

$\mathrm{T}^{*}\left(\mathrm{e}_{10}\right)$

Figure 2: $\mathscr{G}^{*}=\left(T^{*}\left(e_{9}\right), T^{*}\left(e_{10}\right)\right)$.

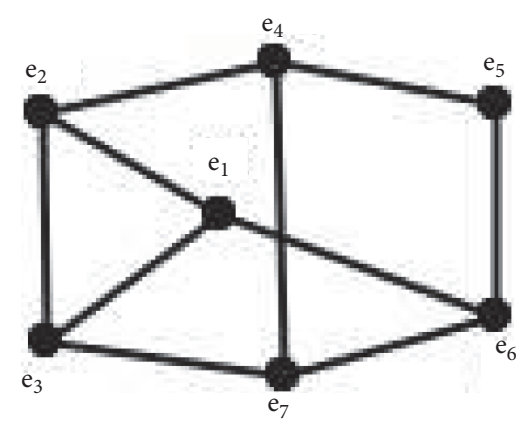

FIGURE 3: Simple graph.

$$
\begin{aligned}
& J^{*}\left(e_{2}\right)=\left\{\left\{e_{1},\left\{e_{1} e_{2}, e_{2} e_{3}, e_{3} e_{1}, e_{1} e_{6}\right\}\right\},\right. \\
& \left.\quad\left\{e_{3},\left\{e_{1} e_{2}, e_{2} e_{3}, e_{3} e_{1}, e_{3} e_{7}\right\}\right\},\left\{e_{4},\left\{e_{4} e_{7}, e_{4} e_{5}, e_{4} e_{2}\right\}\right\}\right\}, \\
& I^{*}\left(e_{5}\right)=\left\{\left\{e_{4},\left\{e_{6}, e_{1}, e_{3}\right\},\left\{e_{6},\left\{e_{3}, e_{4}, e_{2}\right\}\right\}\right\},\right. \\
& J^{*}\left(e_{5}\right)=\left\{\left\{e_{4},\left\{e_{6} e_{1}, e_{1} e_{3}\right\}\right\},\left\{e_{6},\left\{e_{3} e_{2}, e_{4} e_{2}\right\}\right\}\right\},
\end{aligned}
$$

Then $\left[I^{* c}, A\right]$ and $\left[J^{* c}, A\right]$ are as follows:

$$
\begin{aligned}
& I^{* c}\left(e_{2}\right)=\left\{\left\{e_{1},\left\{e_{1}, e_{3}, e_{6}, e_{2}\right\}\right\},\left\{e_{3},\left\{e_{1}, e_{3}, e_{7}, e_{2}\right\}\right\},\right. \\
& \left.\left\{e_{4},\left\{e_{7}, e_{5}, e_{4}, e_{2}\right\}\right\}\right\}, \\
& J^{* c}\left(e_{2}\right)=\left\{\left\{e_{1},\left\{e_{2} e_{6}, e_{6} e_{3}\right\}\right\},\left\{e_{3},\left\{e_{1} e_{7}, e_{2} e_{7}\right\}\right\},\right. \\
& \left.\left\{e_{4},\left\{e_{2} e_{7}, e_{2} e_{5}, e_{7} e_{5}\right\}\right\}\right\}, \\
& I^{* c}\left(e_{5}\right)=\left\{\left\{e_{4},\left\{e_{6}, e_{1}, e_{3}\right\},\left\{e_{6},\left\{e_{3}, e_{4}, e_{2}\right\}\right\}\right\},\right. \\
& J^{* c}\left(e_{5}\right)=\left\{\left\{e_{4},\left\{e_{6} e_{3}\right\}\right\},\left\{e_{6},\left\{e_{3} e_{4}\right\}\right\}\right\} .
\end{aligned}
$$

The complement of $\mathscr{G}^{*}=\left(T^{*}\left(e_{2}\right), T^{*}\left(e_{5}\right)\right)$ is $\mathscr{G}^{* c}=\left(T^{* c}\left(e_{2}\right)\right.$, $\left.T^{* c}\left(e_{5}\right)\right)$ as shown in Figure 4.

Proposition 20. If $\mathscr{G}^{*}$ is a regular $T 2 S G$ of $\mathscr{G}$ then $\mathscr{G}^{* c}$ is a regular T2SG of $\mathscr{G}$.

Proof. Let $\mathscr{G}^{*}$ be a regular T2SG of $\mathscr{G}$. Let $\left(T_{\alpha}, \mathcal{N} \mathscr{B}_{\alpha}\right)$ be a $T 1 S G$ corresponding to $T^{*}(\alpha)$ for all $\alpha \in A$. Then $T_{\alpha}(x) \forall x \in$ $\mathscr{N} \mathscr{B}_{\alpha}$ is a regular subgraph of $\mathscr{G}$. Since complement of a regular graph is regular, $T_{\alpha}^{c}(x) \forall x \in \mathscr{N}_{\mathscr{B}_{\alpha}}$ is a regular subgraph. This implies that T1SG corresponding to $T^{* c}(\alpha)$ for all $\alpha \in A$ is regular T1SG. Hence $\mathscr{G}^{* c}$ is a regular T2SG of $\mathscr{G}$.

Proposition 21. Let $\mathscr{G}$ be a regular graph. Then every T2SG of $\mathscr{G}$ may not be a regular T2SG of $\mathscr{G}$.

Example 22. Consider a regular graph $\mathscr{G}=(X, \mathscr{E})$, where $X=\left\{e_{1}, e_{2}, e_{3}, e_{4}, e_{5}, e_{6}\right\}$ and $\mathscr{E}=\left\{e_{1} e_{2}, e_{3} e_{2}, e_{3} e_{4}, e_{4} e_{5}\right.$, $\left.e_{5} e_{6}, e_{1} e_{6}, e_{1} e_{4}, e_{6} e_{3}, e_{5} e_{2}\right\}$. Let $A=\left\{e_{1}, e_{4}\right\}$. It may be written that $\mathscr{N}_{e_{1}}=\left\{e_{2}, e_{6}, e_{4}\right\}$ and $\mathcal{N} \mathscr{B}_{e_{5}}=\left\{e_{4}, e_{6}, e_{2}\right\}$. Let $\left[I^{*}, A\right]$ and $\left[J^{*}, A\right]$ be two T2SSs over $X$ and $\mathscr{E}$ respectively, such that $I^{*}(x)=\left(I_{x}, \mathcal{N} \mathscr{B}_{x}\right)$ and $J^{*}(x)=\left(J_{x}, \mathcal{N} \mathscr{B}_{x}\right)$ for all $x \in A$. Define $I_{e_{1}}(z)=\{y \in X \mid z \mathscr{R} y \Longleftrightarrow d(z, y) \leq 1\}$, $J_{e_{1}}(z)=\left\{\alpha \beta \in \mathscr{E} \mid\{\alpha, \beta\} \subseteq I_{e_{1}}(z)\right\}$ for all $z \in \mathscr{N} \mathscr{B}_{e_{1}} \subseteq X$, and $I_{e_{5}}(z)=\{y \in X \mid z \mathscr{R} y \Longleftrightarrow d(z, y) \leq 1\}, J_{e_{5}}(z)=\{\alpha \beta \in \mathscr{E} \mid$ $\left.\{\alpha, \beta\} \subseteq I_{e_{5}}(z)\right\}$ for all $z \in \mathscr{N}_{e_{5}} \subseteq X$. Then T2SSs $\left[I^{*}, A\right]$ and $\left[J^{*}, A\right]$ are as follows:

$$
\begin{aligned}
& I^{*}\left(e_{1}\right)=\left\{\left\{e_{2},\left\{e_{2}, e_{1}, e_{5}, e_{3}\right\}\right\},\left\{e_{4},\left\{e_{4}, e_{1}, e_{5}, e_{3}\right\}\right\},\right. \\
& \left.\quad\left\{e_{6},\left\{e_{1}, e_{6}, e_{3}, e_{5}\right\}\right\}\right\}, \\
& J^{*}\left(e_{1}\right)=\left\{\left\{e_{2},\left\{e_{1} e_{2}, e_{2} e_{4}, e_{5} e_{2}\right\}\right\},\left\{e_{4},\left\{e_{5} e_{4}, e_{4} e_{1}, e_{4} e_{3}\right\}\right\},\right. \\
& \left.\quad\left\{e_{6},\left\{e_{5} e_{6}, e_{6} e_{3}, e_{1} e_{6}\right\}\right\}\right\}, \\
& I^{*}\left(e_{5}\right)=\left\{\left\{e_{4},\left\{e_{2}, e_{1}, e_{5}, e_{6}, e_{3}\right\}\right\},\left\{e_{2},\left\{e_{3}, e_{4}, e_{1}, e_{6}, e_{5}\right\}\right\},\right. \\
& \left.\quad\left\{e_{6},\left\{e_{3}, e_{4}, e_{1}, e_{2}, e_{5}\right\}\right\}\right\}, \\
& J^{*}\left(e_{5}\right)=\left\{\left\{e_{4},\left\{e_{1} e_{2}, e_{3} e_{2}, e_{5} e_{6}, e_{1} e_{6}, e_{6} e_{3}, e_{5} e_{2}\right\}\right\},\right. \\
& \quad\left\{e_{6},\left\{e_{1} e_{2}, e_{3} e_{2}, e_{3} e_{4}, e_{4} e_{5}, e_{1} e_{4}, e_{5} e_{2}\right\}\right\}, \\
& \left.\quad\left\{e_{2},\left\{e_{3} e_{4}, e_{4} e_{5}, e_{5} e_{6}, e_{1} e_{6}, e_{1} e_{4}, e_{6} e_{3}\right\}\right\}\right\} .
\end{aligned}
$$

Then $\mathscr{G}^{*}=\left(T^{*}\left(e_{1}\right), T^{*}\left(e_{5}\right)\right)$ is not a regular T2SG of $\mathscr{G}$ as shown in Figure 5.

Definition 23. Let $\mathscr{G}^{*}$ be a T2SG of $\mathscr{G}$. Then $\mathscr{G}^{*}$ is said to be irregular T2SG if T1SG corresponding to $T^{*}(\gamma)$ is an irregular T1SG for all $\gamma \in A$.

Example 24. Consider a graph $\mathscr{G}=(X, \mathscr{E})$ as shown in Figure 6. Let $A=\left\{e_{5}, e_{6}\right\}$. It may be written that $\mathcal{N} \mathscr{B}_{e_{5}}=$ $\left\{e_{1}, e_{6}\right\}$ and $\mathscr{N}_{e_{6}}=\left\{e_{5}, e_{7}\right\}$. Let $\left[I^{*}, A\right]$ and $\left[J^{*}, A\right]$ be 


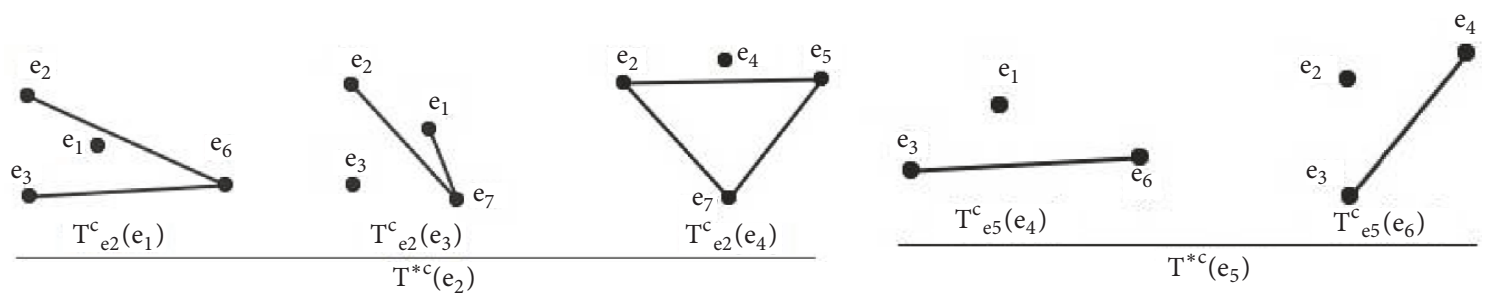

Figure 4: $\mathscr{G}^{* c}=\left(T^{* c}\left(e_{2}\right), T^{* c}\left(e_{5}\right)\right)$.

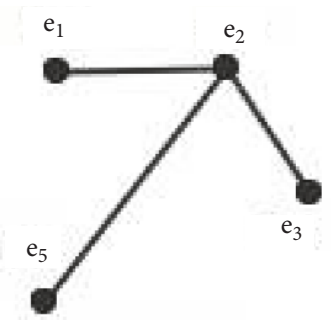

$\mathrm{T}_{\mathrm{el}}\left(\mathrm{e}_{2}\right)$

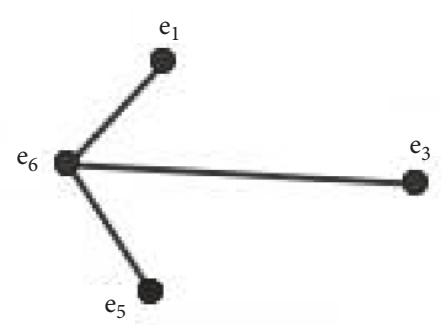

$\mathrm{T}_{\mathrm{el}}\left(\mathrm{e}_{6}\right)$

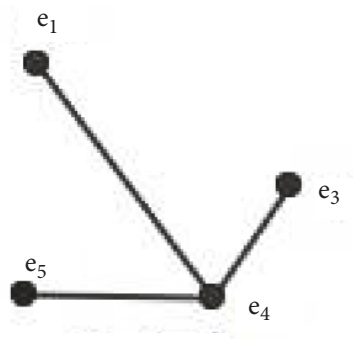

$\mathrm{T}_{\mathrm{el}}\left(\mathrm{e}_{4}\right)$

$\mathrm{T}^{*}\left(\mathrm{e}_{1}\right)$

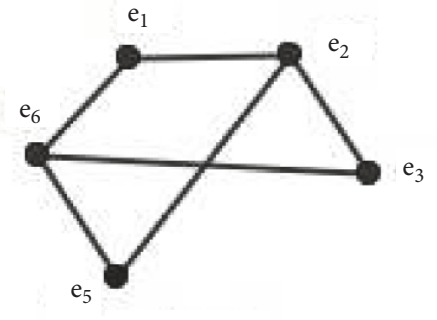

$\mathrm{T}_{\mathrm{e} 5}\left(\mathrm{e}_{4}\right)$

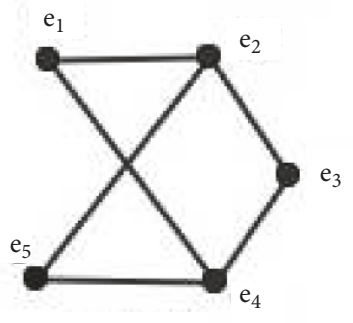

$\mathrm{T}_{\mathrm{e} 5}\left(\mathrm{e}_{6}\right)$

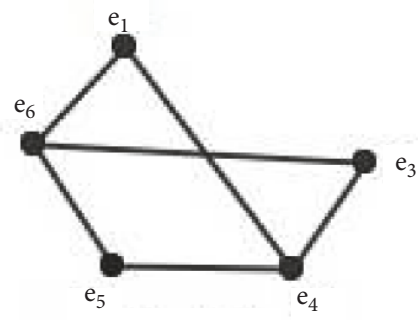

$\mathrm{T}_{\mathrm{e} 5}\left(\mathrm{e}_{2}\right)$

$\mathrm{T}^{*}\left(\mathrm{e}_{5}\right)$

Figure 5: $\mathscr{G}^{*}=\left(T^{*}\left(e_{1}\right), T^{*}\left(e_{5}\right)\right)$.

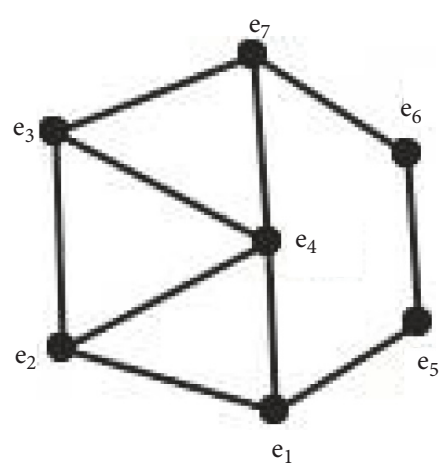

Figure 6: Simple graph.

two T2SSs over $X$ and $\mathscr{E}$ respectively, such that $I^{*}(x)=$ $\left(I_{x}, \mathcal{N} \mathscr{B}_{x}\right)$ and $J^{*}(x)=\left(J_{x}, \mathcal{N} \mathscr{B}_{x}\right)$ for all $x \in A$. Define $I_{e_{5}}(z)=\{y \in X \mid z \mathscr{R} y \Longleftrightarrow d(z, y) \leq 1\}, J_{e_{5}}(z)=\{\alpha \beta \in \mathscr{E} \mid$ $\left.\{\alpha, \beta\} \subseteq I_{e_{5}}(z)\right\}$ for all $z \in \mathcal{N} \mathscr{B}_{e_{5}} \subseteq X$, and $I_{e_{6}}(z)=\{y \in X \mid$ $z \mathscr{R} y \Longleftrightarrow d(z, y) \leq 1\}, J_{e_{6}}(z)=\left\{\alpha \beta \in \mathscr{E} \mid\{\alpha, \beta\} \subseteq I_{e_{6}}(z)\right\}$ for all $z \in \mathcal{N} \mathscr{B}_{e_{6}} \subseteq X$. Then T2SSs $\left[I^{*}, A\right]$ and $\left[J^{*}, A\right]$ are as follows:

$$
\begin{aligned}
& I^{*}\left(e_{5}\right)=\left\{\left\{e_{1},\left\{e_{1}, e_{4}, e_{5}, e_{2}\right\}\right\},\left\{e_{6},\left\{e_{5}, e_{6}, e_{7}\right\}\right\}\right\}, \\
& J^{*}\left(e_{5}\right) \\
& \quad=\left\{\left\{e_{1},\left\{e_{1} e_{2}, e_{2} e_{4}, e_{5} e_{1}, e_{1} e_{4}\right\}\right\},\left\{e_{6},\left\{e_{5} e_{6}, e_{6} e_{7}\right\}\right\}\right\}, \\
& I^{*}\left(e_{6}\right)=\left\{\left\{e_{5},\left\{e_{6}, e_{1}, e_{5}\right\}\right\},\left\{e_{7},\left\{e_{3}, e_{4}, e_{6}, e_{7}\right\}\right\}\right\}, \\
& J^{*}\left(e_{6}\right) \\
& \quad=\left\{\left\{e_{5},\left\{e_{6} e_{1}, e_{5} e_{6}\right\}\right\},\left\{e_{7},\left\{e_{3} e_{4}, e_{7} e_{3}, e_{7} e_{4}, e_{7} e_{6}\right\}\right\}\right\} .
\end{aligned}
$$

Then $\mathscr{G}^{*}=\left(T^{*}\left(e_{6}\right), T^{*}\left(e_{5}\right)\right)$ is an irregular T2SG of $\mathscr{G}$ as shown in Figure 7.

Definition 25. Let $\mathscr{G}^{*}$ be a type 2 soft graph of $\mathscr{G}$. Then $\mathscr{G}^{*}$ is said to be neighborly irregular T1SG if T1SG corresponding to $T^{*}(\gamma)$ is an neighborly irregular T1SG for all $\gamma \in A$. 

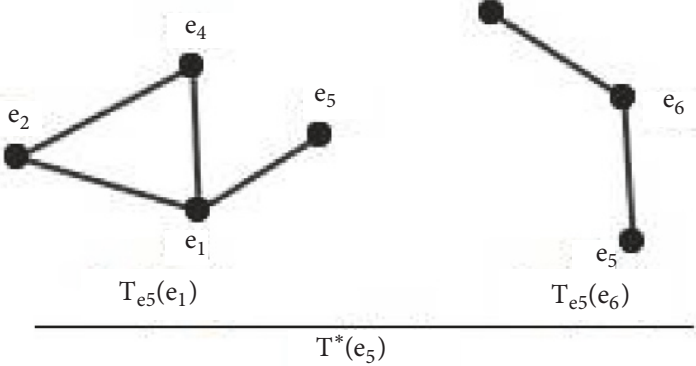

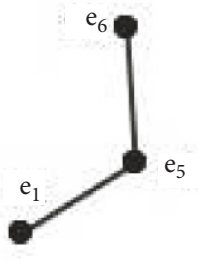

$\mathrm{T}_{\mathrm{e} 6}\left(\mathrm{e}_{5}\right)$

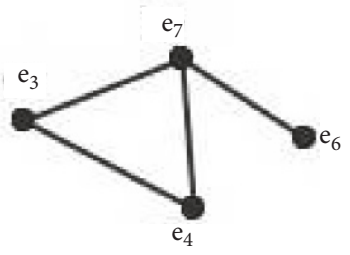

$\mathrm{T}_{\mathrm{e} 6}\left(\mathrm{e}_{7}\right)$

$\mathrm{T}^{*}\left(\mathrm{e}_{6}\right)$

Figure 7: $\mathscr{G}^{*}=\left(T^{*}\left(e_{5}\right), T^{*}\left(e_{6}\right)\right)$.

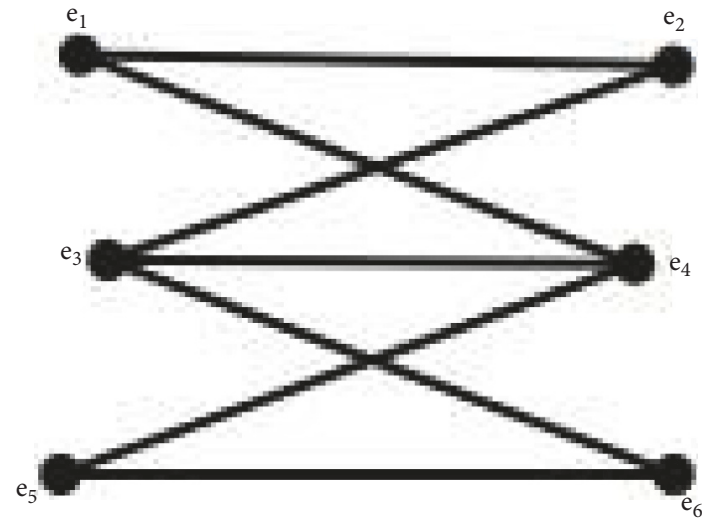

FIGURE 8: Simple graph.

Example 26. Consider a graph $\mathscr{G}=(X, \mathscr{E})$ as shown in Figure 8. Let $A=\left\{e_{1}, e_{6}\right\}$. It may be written that $\mathcal{N} \mathscr{B}_{e_{1}}=$ $\left\{e_{2}, e_{4}\right\}$ and $\mathcal{N} \mathscr{B}_{e_{6}}=\left\{e_{5}, e_{3}\right\}$. Let $\left[I^{*}, A\right]$ and $\left[J^{*}, A\right]$ be two T2SSs over $X$ and $\mathscr{E}$ respectively, such that $I^{*}(x)=$ $\left(I_{x}, \mathcal{N} \mathscr{B}_{x}\right)$ and $J^{*}(x)=\left(J_{x}, \mathcal{N} \mathscr{B}_{x}\right)$ for all $x \in A$. Define $I_{e_{1}}(z)=\{y \in X \mid z \mathscr{R} y \Longleftrightarrow d(z, y) \leq 1\}, J_{e_{1}}(z)=\{\alpha \beta \in \mathscr{E} \mid$ $\left.\{\alpha, \beta\} \subseteq I_{e_{1}}(z)\right\}$ for all $z \in \mathcal{N} \mathscr{B}_{e_{1}} \subseteq X$, and $I_{e_{6}}(z)=\{y \in X \mid$ $z \mathscr{R} y \Longleftrightarrow d(z, y) \leq 1\}, J_{e_{6}}(z)=\left\{\alpha \beta \in \mathscr{E} \mid\{\alpha, \beta\} \subseteq I_{e_{6}}(z)\right\}$ for all $z \in \mathcal{N} \mathscr{B}_{e_{6}} \subseteq X$. Then T2SSs $\left[I^{*}, A\right]$ and $\left[J^{*}, A\right]$ are as in the following; $I^{*}\left(e_{1}\right)=\left\{\left\{e_{2},\left\{e_{1}, e_{3}, e_{2}\right\}\right\},\left\{e_{4},\left\{e_{4}, e_{1}, e_{3}, e_{5}\right\}\right\}\right\}$, $J^{*}\left(e_{1}\right)=\left\{\left\{e_{2},\left\{e_{1} e_{2}, e_{2} e_{3}\right\}\right\},\left\{e_{4},\left\{e_{4} e_{3}, e_{4} e_{5}, e_{4} e_{2}\right\}\right\}\right\}, I^{*}\left(e_{6}\right)=$ $\left\{\left\{e_{3},\left\{e_{3}, e_{2}, e_{4}, e_{6}\right\}\right\},\left\{e_{5},\left\{e_{5}, e_{4}, e_{6}\right\}\right\}\right\}, J^{*}\left(e_{6}\right)=\left\{\left\{e_{3},\left\{e_{4} e_{3}, e_{3} e_{6}\right.\right.\right.$, $\left.\left.\left.e_{3} e_{2}\right\}\right\},\left\{e_{5},\left\{e_{5} e_{4}, e_{5} e_{6}\right\}\right\}\right\}$, Then $\mathscr{G}^{*}=\left(T^{*}\left(e_{1}\right), T^{*}\left(e_{6}\right)\right)$ is a neighborly irregular T2SG of $\mathscr{G}$ as shown in Figure 9.

Definition 27. Let $\mathscr{G}^{*}$ be a T2SG of a simple graph $\mathscr{G}$. Let $\left(T_{\alpha}, \mathcal{N} \mathscr{B}_{\alpha}\right)$ be a T1SG corresponding to $T^{*}(\alpha)$ for all $\alpha \in A$. An ede $u v$ in $\mathscr{G}^{*}$ is said to be a type 2 soft bridge if its removal disconnect the subgraph $T_{\alpha}(x), \forall x \in \mathcal{N} \mathscr{B}_{\alpha}$.

Definition 28. Let $\mathscr{G}^{*}$ be a T2SG of a simple graph $\mathscr{G}$. Let $\left(T_{\alpha}, \mathcal{N} \mathscr{B}_{\alpha}\right)$ be a T1SG corresponding to $T^{*}(\alpha)$ for all $\alpha \in A$. An vertex $z$ in $\mathscr{G}^{*}$ is said to be a type 2 soft cut-vertex if its removal disconnect the subgraph $T_{\alpha}(x), \forall x \in \mathcal{N} \mathscr{B}_{\alpha}$.
Example 29. Consider T2SG $\mathscr{G}^{*}$ defined in Example 24. In the Figure 7, type 2 soft bridges of $\mathscr{G}^{*}$ are $e_{1} e_{5}$ in $T_{e_{5}}\left(e_{1}\right)$, $e_{7} e_{6}, e_{6} e_{5}$ in $T_{e_{5}}\left(e_{6}\right), e_{5} e_{6}, e_{5} e_{1}$ in $T_{e_{6}}\left(e_{5}\right)$ and $e_{6} e_{7}$ in $T_{e_{6}}\left(e_{7}\right)$. Moreover, type 2 cut-vertices of $\mathscr{G}^{*}$ are $e_{1}$ in $T_{e_{5}}\left(e_{1}\right), e_{5}$ in $T_{e_{5}}\left(e_{6}\right), e_{6}$ in $T_{e_{6}}\left(e_{5}\right)$ and $e_{7}$ in $T_{e_{6}}\left(e_{7}\right)$.

Definition 30. Let $\mathscr{G}^{*}=\left(\mathscr{G}, I^{*}, J^{*}, A, \mathcal{N} \mathscr{B}_{A}\right)$ be a $T 2 S G$ of $\mathscr{G}$. Then $\mathscr{G}^{*}$ is said to be a type 2 soft tree (briefly, T2ST) if T1SG corresponding to every $T^{*}(\gamma)$ is a T1ST for all $\gamma \in A$.

Example 31. Consider a graph $\mathscr{G}=(X, \mathscr{E})$ as shown in Figure 10. Let $A=\{c, f\} \subset X$ and $\mathscr{N}_{\mathscr{B}_{c}}=\{b, d\}, \mathcal{N} \mathscr{B}_{f}=$ $\{e, g\}$. Let $\left[I^{*}, A\right]$ and $\left[J^{*}, A\right]$ be two neighbor-induced T2SSs over $X$ and $\mathscr{E}$ respectively, such that $I^{*}(x)=\left(I_{x}, \mathcal{N} \mathscr{B}_{x}\right)$ and $J^{*}(x)=\left(J_{x}, \mathcal{N} \mathscr{B}_{x}\right)$ for all $x \in A$. We define $I_{c}(z)=\{y \in X \mid$ $z \mathscr{R} y \Longleftrightarrow d(z, y)=\operatorname{rad}(G)\}, J_{c}(z)=\{\alpha \beta \in \mathscr{E} \mid\{\alpha, \beta\} \subseteq$ $\left.I_{c}(z)\right\}$ for all $z \in \mathscr{N}_{c} \subseteq X$ and $I_{f}(z)=\{y \in X \mid z \mathscr{R} y \Longleftrightarrow$ $d(z, y)=\operatorname{rad}(G)\}, J_{f}(z)=\left\{\alpha \beta \in \mathscr{E} \mid\{\alpha, \beta\} \subseteq I_{f}(z)\right\}$ for all $z \in \mathcal{N} \mathscr{B}_{f} \subseteq X$. Then T2SSs $\left[I^{*}, A\right]$ and $\left[J^{*}, A\right]$ are as in the following $I^{*}(c)=\{\{b,\{g, e\}\},\{d,\{a, g, f\}\}\}, J^{*}(c)=$ $\{\{b,\{g e\}\},\{d,\{a g, g f\}\}\}, I^{*}(f)=\{\{g,\{d, b\}\},\{e,\{a, b, c\}\}\}$, $J^{*}(f)=\{\{g,\{b d\}\},\{e,\{a b, b c\}\}\}$. Therefore, $\mathscr{G}^{*}=\left(T^{*}(c)\right.$, $\left.T^{*}(f)\right)$ is a T2ST of $\mathscr{G}$ as shown in Figure 11. It is also called $V N$-type 2 soft tree.

Hence, $\mathscr{G}^{*}=\left(T^{*}(c), T^{*}(f)\right)$ is a T2SG of $\mathscr{G}$. It is also called $V N$-type 2 soft graph. It may be written that $\mathscr{P}=$ $\bigcup_{x \in A} \mathcal{N} \mathscr{B}_{x}=\{b, d, e, g\}$. We may symbolize $\alpha \in \mathcal{N} \mathscr{B}_{x}$, as $(x \mapsto \alpha)$ and denote a set of associations of $A$, as $(A \mapsto \mathscr{P})=$ $\left\{(x \mapsto \alpha) \mid \alpha \in \mathcal{N} \mathscr{B}_{x}\right\}$. Then tabular representation of T2ST is given in Table 1 .

Theorem 32. Let $\left(T_{\gamma}, \mathcal{N} \mathscr{B}_{\gamma}\right)$ be a T1SS corresponding to $T^{*}(\gamma) \forall \gamma \in A$. Let $T_{\gamma}(x) \forall x \in \mathcal{N} \mathscr{B}_{\gamma}$ be subgraph with $n \geq 3$ vertices of $\mathscr{G}$ and $\mathscr{G}^{*}$ a T2SG of $\mathscr{G}$. Then $\mathscr{G}^{*}$ is not a complete T2SG of $\mathscr{G}$.

Proof. Let $\left(T_{\gamma}, \mathcal{N} \mathscr{B}_{\gamma}\right)$ be a $T 1 S S$ corresponding to $T^{*}(\gamma) \forall \gamma \in$ $A$. Suppose on the contrary that $\mathscr{G}$ is a complete $T 2 S G$, then every subgraph $T_{\gamma}(x)$, for all $x \in \mathcal{N} \mathscr{B}_{\gamma}$ is complete. Suppose $\alpha, \beta$ be arbitrary nodes of $\left(T_{\gamma}(x)\right.$ and they are connected by an edge $\alpha \beta$. Since $T_{\gamma}(x)$ is subgraph with $n \geq 3$ vertices of $\mathscr{G}$, then we can always find at least one vertex $\eta$ which is connected to $\alpha$ by an edge $\alpha \eta$ and to $\beta$ by an edge $\beta \eta$, 

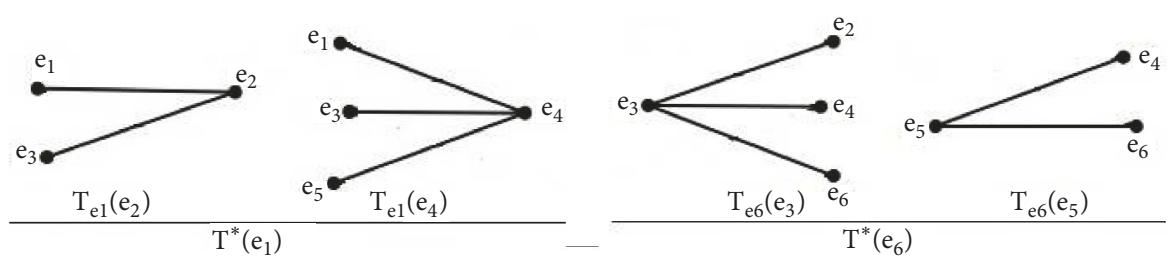

Figure 9: $\mathscr{G}^{*}=\left(T^{*}\left(e_{1}\right), T^{*}\left(e_{6}\right)\right)$.

TABLE 1: Tabular representation of a type 2 soft tree.

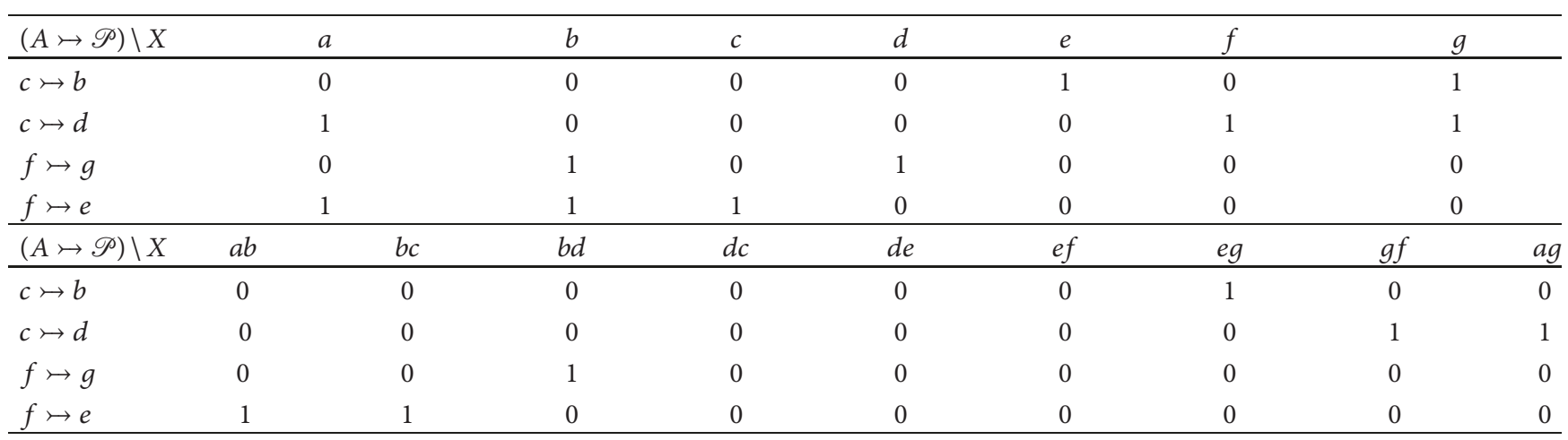

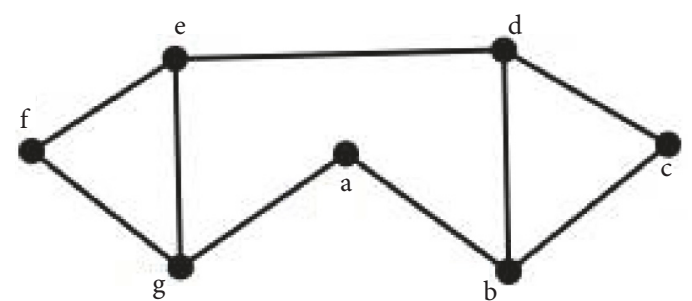

FIGURE 10: Simple graph.

because $T_{\gamma}(x)$ is a complete graph. Then there exists a cycle $\alpha \beta \eta$. Therefore, $T_{\gamma}(x) \forall x \in \mathcal{N} \mathscr{B}_{\gamma}$ cannot be a $T 1 S T$ which contradicts the fact that $T_{\gamma}(x)$ is a connected subgraph of T2SG. Hence, $\mathscr{G}^{*}$ cannot be a complete T2SG.

Definition 33. Let $\mathscr{G}^{*}$ be a $T 2 S G$ and $\left(T_{\gamma}, \mathcal{N} \mathscr{B}_{\gamma}\right)$ be a $T 1 S T$ corresponding to $T^{*}(\gamma)$ for any $\gamma \in A$. Then $\mathscr{G}^{*}$ is called type 2 soft forest if $T_{\gamma}(x)$ consists of more than one disconnected tree for all $x \in \mathcal{N} \mathscr{B}_{\gamma}$.

Definition 34. Let $\mathscr{G}^{*}$ be a T2SG of $\mathscr{G}$. Then $\mathscr{G}^{*}$ is said to be a type 2 soft cycle (briefly, T2SC) if T1SG corresponding to $T(\gamma)$ is a type 1 soft cycle of $\mathscr{G}$ for each $\gamma \in A$.

Example 35. Consider a simple graph $\mathscr{G}=(X, \mathscr{E})$, where $X=$ $\left\{e_{1}, e_{2}, e_{3}, e_{4}, e_{5}, e_{6}, e_{7}, e_{8}, e_{9}, e_{10}\right\}$ and $\mathscr{E}=\left\{e_{1} e_{2}, e_{2} e_{3}, e_{3} e_{1}\right.$, $\left.e_{3} e_{4}, e_{5} e_{3}, e_{5} e_{6}, e_{6} e_{4}, e_{7} e_{6}, e_{7} e_{9}, e_{7} e_{8}, e_{9} e_{10}, e_{8} e_{10}\right\}$. Let $A=$ $\left\{e_{9}, e_{8}\right\} \subset X$. It may be written that $\mathcal{N}_{e_{9}}=\left\{e_{10}, e_{7}\right\}$ and $\mathcal{N} \mathscr{B}_{e_{8}}=\left\{e_{7}, e_{10}\right\}$. Let $\left[I^{*}, A\right]$ and $\left[J^{*}, A\right]$ be two T2SSs over $X$ and $\mathscr{E}$, respectively, such that $I^{*}(x)=\left(I_{x}, \mathcal{N} \mathscr{B}_{x}\right)$ and $J^{*}(x)=$
$\left(J_{x}, \mathcal{N} \mathscr{B}_{x}\right)$ for all $x \in A$. Define $I_{e_{9}}(z)=\{y \in X \mid z \mathscr{R} y \Longleftrightarrow$ $5 \geq d(z, y) \geq 3\}, J_{e_{9}}(z)=\left\{\alpha \beta \in \mathscr{E} \mid\{\alpha, \beta\} \subseteq I_{e_{9}}(z)\right\}$ for all $z \in \mathscr{N}_{e_{9}} \subseteq X$, and $I_{e_{8}}(z)=\{y \in X \mid z \mathscr{R} y \Longleftrightarrow$ $5 \geq d(z, y) \geq 3\}, J_{e_{8}}(z)=\left\{\alpha \beta \in \mathscr{E} \mid\{\alpha, \beta\} \subseteq I_{e_{8}}(z)\right\}$ for all $z \in \mathcal{N}_{e_{8}} \subseteq X$. Then T2SSs $\left[I^{*}, A\right]$ and $\left[J^{*}, A\right]$ are as follows:

$$
\begin{aligned}
& I^{*}\left(e_{9}\right)=\left\{\left\{e_{7},\left\{e_{2}, e_{3}, e_{1}\right\},\left\{e_{10},\left\{e_{5}, e_{3}, e_{4}, e_{6}\right\}\right\},\right.\right. \\
& J^{*}\left(e_{9}\right)=\left\{\left\{e_{10},\left\{e_{2} e_{3}, e_{3} e_{1}, e_{2} e_{1}\right\}\right\},\right. \\
& \left.\left\{e_{10},\left\{e_{4} e_{3}, e_{3} e_{5}, e_{6} e_{5}, e_{6} e_{4}\right\}\right\}\right\}, \\
& I^{*}\left(e_{8}\right)=\left\{\left\{e_{7},\left\{e_{2}, e_{3}, e_{1}\right\},\left\{e_{10},\left\{e_{5}, e_{3}, e_{4}, e_{6}\right\}\right\}\right\},\right. \\
& J^{*}\left(e_{8}\right)=\left\{\left\{e_{10},\left\{e_{2} e_{3}, e_{3} e_{1}, e_{2} e_{1}\right\}\right\},\right. \\
& \left.\left\{e_{10},\left\{e_{4} e_{3}, e_{3} e_{5}, e_{6} e_{5}, e_{6} e_{4}\right\}\right\}\right\} .
\end{aligned}
$$

One can check that $\mathscr{G}^{*}=\left(T^{*}\left(e_{4}\right), T^{*}\left(e_{8}\right)\right)$ is a T2SC of $\mathscr{G}$ as shown in Figure 12. It is also called VN-type 2 soft cycle.

Theorem 36. If $\mathscr{G}^{*}$ is a T2SC of $\mathscr{G}$ then $\mathscr{G}^{*}$ is not a T2ST of $\mathscr{G}$.

Proof. Let $\mathscr{G}^{*}$ be a T2SC of $\mathscr{G}$. Let $\left(T_{\gamma}, \mathcal{N} \mathscr{B}_{\gamma}\right)$ be a type 1 soft cycle corresponding to $T^{*}(\gamma) \forall \gamma \in A$. By definition, tree does not contain cycle. Then $T_{\gamma}(x)$ is not a tree for all $x \in \mathcal{N} \mathscr{B}_{\gamma}$, so that $\left(T_{\gamma}, \mathcal{N} \mathscr{B}_{\gamma}\right)$ is not a type 1 soft tree. Hence $\mathscr{G}^{*}$ is not a T2ST of $\mathscr{G}$.

The converse of above theorem is not true in general; i.e., if $\mathscr{G}^{*}$ is not a T2ST then $\mathscr{G}$ need not be a T2SC. The following example illustrates it. 

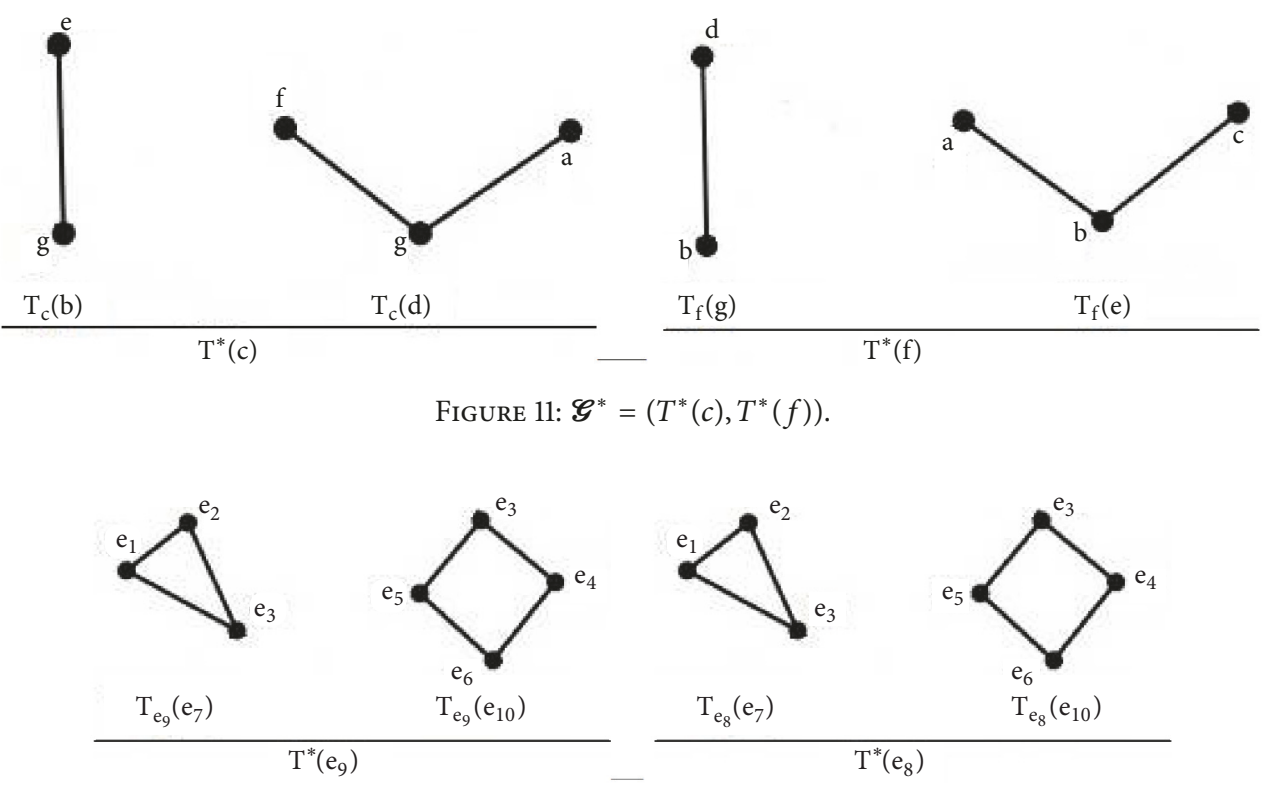

Figure 12: $\mathscr{G}^{*}=\left(T^{*}\left(e_{9}\right), T^{*}\left(e_{8}\right)\right)$.

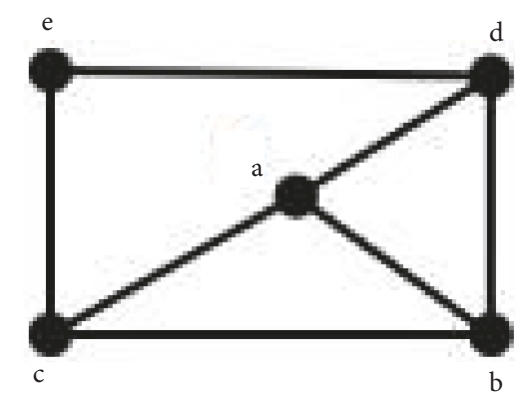

Figure 13: Simple graph.

Example 37. Consider a graph $\mathscr{G}=(X, \mathscr{E})$ as shown in Figure 13.

Let $=\{a, b\} \quad \subset \quad X$. Then $\mathscr{N}_{\mathscr{B}_{a}}=\{b, c, d\}, \mathscr{N}_{\mathscr{B}_{b}}=$ $\{a, c, d\}$. Let $\left[I^{*}, A\right]$ and $\left[J^{*}, A\right]$ be two T2SSs over $X$ and $\mathscr{E}$, respectively, such that $I^{*}(x)=\left(I_{x}, \mathcal{N} \mathscr{B}_{x}\right)$ and $J^{*}(x)=$ $\left(J_{x}, \mathscr{N}_{x}\right)$ for all $x \in A$. Let $I_{a}(\alpha)=\{y \in X \mid \alpha \mathscr{R} y \Longleftrightarrow$ $d(\alpha, y) \leq 1\}, J_{a}(\alpha)=\left\{u v \in \mathscr{E} \mid\{u, v\} \subseteq I_{a}(\alpha)\right\} \forall \alpha \in \mathscr{N}_{a}$ and $I_{b}(\beta)=\left\{y^{\prime} \in X \mid \beta \mathscr{R} y^{\prime} \Longleftrightarrow d\left(\beta, y^{\prime}\right)=1\right\}, J_{b}(\beta)=$ $\left\{u^{\prime} v^{\prime} \in \mathscr{E} \mid\left\{u^{\prime}, v^{\prime}\right\} \subseteq I_{b}(\beta)\right\} \forall \beta \in \mathscr{N} \mathscr{B}_{b}$. Then

$$
\begin{aligned}
& I^{*}(a)=\{\{b,\{a, b, d, c\}\},\{c,\{a, b, c, e\}\},\{d,\{a, b, d, e\}\}\}, \\
& J^{*}(a)=\{\{b,\{a b, a d, a c, b c, b d\}\},\{c,\{a b, a c, c e, b c\}\}, \\
& \{d,\{a b, a d, d e, d b\}\}\}, \\
& I^{*}(b)=\{\{a,\{b, c, d\}\},\{c,\{a, b, e\}\},\{d,\{a, b, e\}\}\}, \\
& J^{*}(b)=\{\{a,\{b c, b d\}\},\{c,\{a b\}\},\{d,\{a b\}\}\}
\end{aligned}
$$

Figure 14 shows the respective T1SGs corresponding to $T^{*}(a)=\left(I^{*}(a), J^{*}(a)\right)$ and $T^{*}(b)=\left(I^{*}(b), J^{*}(b)\right)$ respectively. One can check that $T_{a}(b)=\left(I_{a}(b), J_{a}(b)\right), T_{a}(c)=$
$\left(I_{a}(c), J_{a}(c)\right), T_{a}(d)=\left(I_{a}(d), J_{a}(d)\right), T_{b}(c)=\left(I_{b}(c), J_{b}(c)\right)$ and $\mathrm{T}_{b}(d)=\left(I_{b}(d), J_{b}(d)\right)$ are not trees. This implies that $\mathscr{G}^{*}=\left(T^{*}(a), T^{*}(b)\right)$ is not a T2ST of $\mathscr{G}$. But $\mathscr{G}^{*}$ is not a T2SC of $\mathscr{G}$.

Proposition 38. Every T2SC of $\mathscr{G}$ is a regular T2SG of $\mathscr{G}$.

Proof. Suppose that $\mathscr{G}^{*}$ is a T2SC. Let $\left(T_{\gamma}, \mathcal{N} \mathscr{B}_{\gamma}\right)$ be a T1SC corresponding to $T^{*}(\gamma)$ for any $\gamma \in A$. Then $T_{\gamma}(x)$ is a cycle for all $x \in \mathscr{N} \mathscr{B}_{\alpha}$. Since cycle is closed path and each vertex has degree 2 , this implies that $T_{\gamma}(x)$ is a regular graph for all $x \in \mathscr{N} \mathscr{B}_{\gamma}$. Therefore $\left(T_{\gamma}, \mathcal{N} \mathscr{B}_{\gamma}\right)$ is regular T1SG. Since $\gamma$ was taken to be arbitrary, thus it holds for all $\gamma \in A$. Hence $\mathscr{G}^{*}$ is a regular $T 2 S G$ of $\mathscr{G}$.

\section{Operations on Type 2 Soft Trees}

In this section, we present type 2 soft subtree of $T 2 S T$, union, intersection, OR operation, and AND operation of T2ST.

Definition 39. Let $\mathscr{G}_{1}^{*}=\left\langle I_{1}^{*}, J_{1}^{*}, A_{1}\right\rangle$ and $\mathscr{G}_{2}^{*}=\left\langle I_{2}^{*}, J_{2}^{*}, A_{2}\right\rangle$ be two T2STs of $\mathscr{G}$. Then $\mathscr{G}_{2}^{*}$ is a type 2 soft subtree of $\mathscr{G}_{1}^{*}$ if

(i) $A_{2} \subseteq A_{1}$,

(ii) for each $x \in A_{2}, T 1 S T$ corresponding to $T_{2}^{*}(x)=$ $\left(I_{2}^{*}(x), J_{2}^{*}(x)\right)$ is a type 1 soft subtree of T1ST corresponding to $T_{1}^{*}(x)=\left(I_{1}^{*}(x), J_{1}^{*}(x)\right)$.

Example 40. Consider a simple graph $\mathscr{G}=(X, \mathscr{E})$, where $X=\left\{e_{1}, e_{2}, e_{3}, e_{4}, e_{5}, e_{6}, e_{7}\right\}$ and $\mathscr{E}=\left\{e_{1} e_{2}, e_{2} e_{3}\right.$, $\left.e_{3} e_{4}, e_{5} e_{4}, e_{5} e_{6}, e_{6} e_{7}, e_{7} e_{1}\right\}$. Let $A=\left\{e_{2}, e_{4}\right\}, B=\left\{e_{2}, e_{4}, e_{7}\right\}$. It may be written that $\mathscr{N}_{e_{2}}=\left\{e_{3}, e_{1}\right\}, \mathcal{N}_{e_{4}}=\left\{e_{3}, e_{5}\right\}$ and $\mathcal{N} \mathscr{B}_{e_{7}}=\left\{e_{1}, e_{6}\right\}$.

Let $\left[I^{*}, A\right]$ and $\left[J^{*}, A\right]$ be two T2SSs over $X$ and $\mathscr{E}$, respectively, such that $I^{*}(x)=\left(I_{x}, \mathcal{N} \mathscr{B}_{x}\right)$ and $J^{*}(x)=$ $\left(J_{x}, \mathscr{N} \mathscr{B}_{x}\right)$ for all $x \in A$. Define $I_{e_{2}}(z)=\{y \in X \mid z \mathscr{R} y \Longleftrightarrow$ 


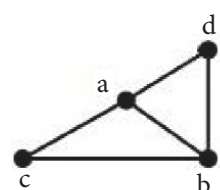

$\mathrm{T}_{\mathrm{a}}(\mathrm{b})$

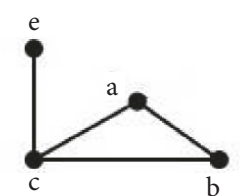

$\mathrm{T}_{\mathrm{a}}(\mathrm{c})$

$\mathrm{T}^{*}$ (a)

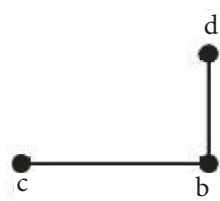

$\mathrm{T}_{\mathrm{b}}(\mathrm{a})$

$\mathrm{T}_{\mathrm{b}}(\mathrm{c})$

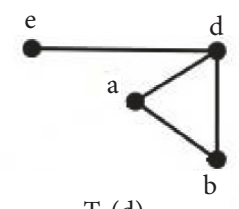

$\mathrm{T}_{\mathrm{a}}(\mathrm{d})$

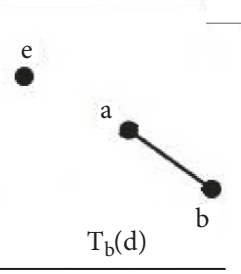

$\mathrm{T}^{*}(\mathrm{~b})$

Figure 14: $\mathscr{G}^{*}=\left(T^{*}(a), T^{*}(b)\right)$.

$d(z, y) \leq 1\}, J_{e_{1}}(z)=\left\{\alpha \beta \in \mathscr{E} \mid\{\alpha, \beta\} \subseteq I_{e_{1}}(z)\right\}$ for all $z \in \mathscr{N} \mathscr{B}_{e_{2}} \subseteq X, I_{e_{4}}(z)=\{y \in X \mid z \mathscr{R} y \Longleftrightarrow d(z, y) \leq 1\}$, $J_{e_{4}}(z)=\left\{\alpha \beta \in \mathscr{E} \mid\{\alpha, \beta\} \subseteq I_{e_{4}}(z)\right\}$ for all $z \in \mathcal{N} \mathscr{B}_{e_{4}} \subseteq X$. Then T2SSs $\left[I^{*}, A\right]$ and $\left[J^{*}, A\right]$ are as follows:

$$
\begin{aligned}
& I^{*}\left(e_{2}\right)=\left\{\left\{e_{1},\left\{e_{1}, e_{2}, e_{7}\right\}\right\},\left\{e_{3},\left\{e_{3}, e_{2}, e_{4}\right\}\right\}\right\}, \\
& J^{*}\left(e_{2}\right)=\left\{\left\{e_{1},\left\{e_{1} e_{2}, e_{7} e_{1}\right\}\right\},\left\{e_{3},\left\{e_{2} e_{3}, e_{3} e_{4}\right\}\right\}\right\}, \\
& I^{*}\left(e_{4}\right)=\left\{\left\{e_{3},\left\{e_{4}, e_{2}, e_{3}\right\}\right\},\left\{e_{5},\left\{e_{6}, e_{5}, e_{4}\right\}\right\}\right\}, \\
& J^{*}\left(e_{4}\right)=\left\{\left\{e_{3},\left\{e_{3} e_{4}, e_{3} e_{2}\right\}\right\},\left\{e_{5},\left\{e_{6} e_{5}, e_{5} e_{4}\right\}\right\}\right\} .
\end{aligned}
$$

Then $\mathscr{G}^{*}=\left(T^{*}\left(e_{2}\right), T^{*}\left(e_{4}\right)\right)$ is a T2ST of $\mathscr{G}$ as shown in Figure 15

Let $\left[I^{\prime *}, B\right]$ and $\left[J^{\prime *}, B\right]$ be two T2SSs over $X$ and $\mathscr{E}$, respectively, such that $I^{\prime *}(x)=\left(J_{x}^{\prime}, \mathcal{N} \mathscr{B}_{x}\right)$ and $J^{\prime *}(x)=$ $\left(J_{x}^{\prime}, \mathcal{N} \mathscr{B}_{x}\right)$ for all $x \in B$. Define

$$
\begin{aligned}
& I_{e_{2}}^{\prime}(z)=\{y \in X \mid z \mathscr{R} y \Longleftrightarrow d(z, y) \leq 1\}, \\
& J_{e_{2}}^{\prime}(z)=\left\{\alpha \beta \in \mathscr{E} \mid\{\alpha, \beta\} \subseteq I_{e_{2}}^{\prime}(z)\right\}
\end{aligned}
$$
for all $z \in \mathcal{N} \mathscr{B}_{e_{2}} \subseteq X$,

$$
\begin{aligned}
& I_{e_{4}}^{\prime}(z)=\{y \in X \mid z \mathscr{R} y \Longleftrightarrow d(z, y) \leq 2\}, \\
& J_{e_{4}}^{\prime}(z)=\left\{\alpha \beta \in \mathscr{E} \mid\{\alpha, \beta\} \subseteq I_{e_{4}}^{\prime}(z)\right\}
\end{aligned}
$$

$$
\text { for all } z \in \mathcal{N} \mathscr{B}_{e_{4}} \subseteq X,
$$

$$
\begin{aligned}
& I_{e_{7}}^{\prime}(z)=\{y \in X \mid z \mathscr{R} y \Longleftrightarrow d(z, y) \leq 1\}, \\
& J_{e_{7}}^{\prime}(z)=\left\{\alpha \beta \in \mathscr{E} \mid\{\alpha, \beta\} \subseteq I_{e_{7}}^{\prime}(z)\right\}
\end{aligned}
$$

$$
\text { for all } z \in \mathcal{N} \mathscr{B}_{e_{7}} \subseteq X \text {. }
$$

Then T2SS $\left[I^{\prime *}, B\right]$ and $\left[J^{\prime *}, B\right]$ are as follows:

$$
\begin{aligned}
& I^{\prime *}\left(e_{2}\right)=\left\{\left\{e_{1},\left\{e_{1}, e_{2}, e_{7}\right\}\right\},\left\{e_{3},\left\{e_{3}, e_{2}, e_{4}\right\}\right\}\right\}, \\
& J^{\prime *}\left(e_{2}\right)=\left\{\left\{e_{1},\left\{e_{1} e_{2}, e_{7} e_{1}\right\}\right\},\left\{e_{3},\left\{e_{2} e_{3}, e_{3} e_{4}\right\}\right\}\right\},
\end{aligned}
$$

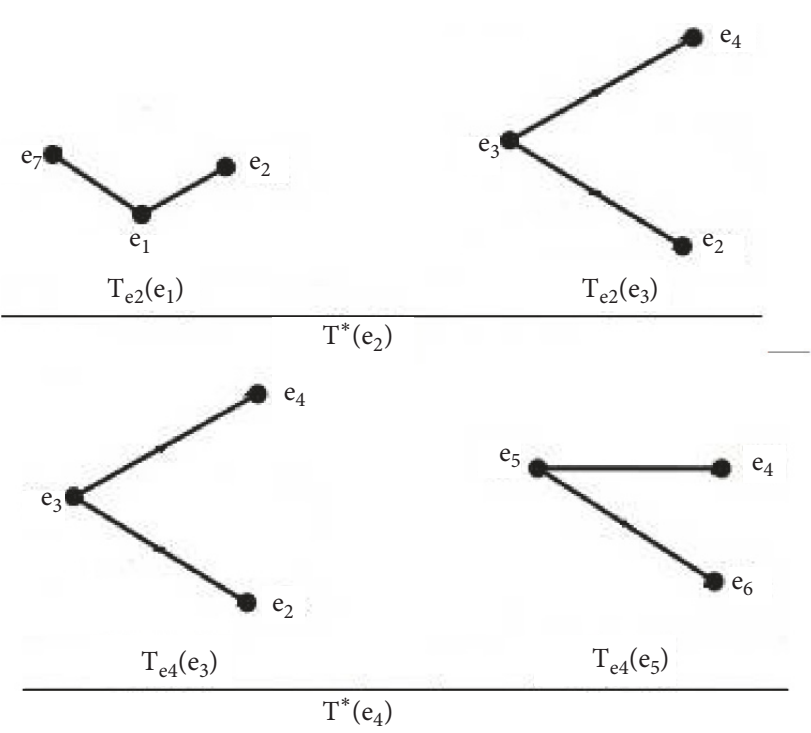

Figure 15: $\mathscr{G}^{*}=\left(T^{*}\left(e_{2}\right), T^{*}\left(e_{4}\right)\right)$.

$$
\begin{aligned}
& I^{\prime *}\left(e_{4}\right)=\left\{\left\{e_{3},\left\{e_{4}, e_{2}, e_{3}, e_{1}, e_{5}\right\}\right\},\right. \\
& \left.\left\{e_{5},\left\{e_{6}, e_{5}, e_{4}, e_{7}, e_{3}\right\}\right\}\right\}, \\
& J^{\prime *}\left(e_{4}\right)=\left\{\left\{e_{3},\left\{e_{1} e_{2}, e_{2} e_{3}, e_{3} e_{4}, e_{5} e_{4}\right\}\right\},\right. \\
& \left.\quad\left\{e_{5},\left\{e_{3} e_{4}, e_{4} e_{5}, e_{5} e_{6}, e_{6} e_{7}\right\}\right\}\right\}, \\
& I^{\prime *}\left(e_{7}\right)=\left\{\left\{e_{1},\left\{e_{1}, e_{2}, e_{7}\right\}\right\},\left\{e_{6},\left\{e_{5}, e_{6}, e_{7}\right\}\right\}\right\}, \\
& J^{\prime *}\left(e_{7}\right)=\left\{\left\{e_{1},\left\{e_{1} e_{2}, e_{7} e_{1}\right\}\right\},\left\{e_{6},\left\{e_{5} e_{6}, e_{6} e_{7}\right\}\right\}\right\},
\end{aligned}
$$

Then $\mathscr{G}^{\prime *}=\left(T^{\prime *}\left(e_{2}\right), T^{* *}\left(e_{4}\right), T^{\prime *}\left(e_{7}\right)\right)$ is a $T 2 S T$ of $\mathscr{G}$ as shown in Figure 16.

One can check that $A \subset B$ and $T^{*}\left(e_{2}\right) \widetilde{\subseteq} T^{* *}\left(e_{2}\right)$, $T^{*}\left(e_{4}\right) \widetilde{\subseteq} T^{\prime *}\left(e_{4}\right)$. Hence $\mathscr{G}^{*}$ is a type 2 subtree of $\mathscr{G}^{\prime *}$.

Theorem 41. Let $\mathscr{G}_{1}^{*}=\left\langle I_{1}^{*}, J_{1}^{*}, A_{1}\right\rangle$ and $\mathscr{G}_{2}^{*}=\left\langle I_{2}^{*}, J_{2}^{*}, A_{2}\right\rangle$ be two T2STs of $\mathscr{G}$. Then $\mathscr{G}_{2}^{*}$ is a type 2 soft subtree of $\mathscr{G}_{1}^{*}$ if and only if $I_{2}^{*}(x) \widetilde{\subseteq} I_{1}^{*}(x)$ and $J_{2}^{*}(x) \widetilde{\subseteq} J_{1}^{*}(x)$ for all $x \in A_{2}$.

Proof. Suppose $\mathscr{G}_{2}^{*}$ is a type 2 soft subtree of $\mathscr{G}_{1}^{*}$. Then, by the definition of type 2 soft subtree,

(i) $A_{2} \subseteq A_{1}$,

(ii) For each $x \in A_{2}, T 1 S T$ corresponding to $T_{2}^{*}(x)=$ $\left(I_{2}^{*}(x), J_{2}^{*}(x)\right)$ is a type 1 soft subtree of T1ST corresponding to $T_{1}^{*}(x)=\left(I_{1}^{*}(x), J_{1}^{*}(x)\right)$.

Since T1ST corresponding to $T_{2}^{*}(x)$ is a type 1 soft subtree of T1ST corresponding to $T_{1}^{*}(x)$ for all $x \in A_{2}$. Then $I_{2}^{*}(x) \widetilde{\widetilde{c}} I_{1}^{*}(x)$ and $J_{2}^{*}(x) \widetilde{\subseteq} J_{1}^{*}(x)$ for all $x \in A_{2}$.

Conversely, given that $I_{2}^{*}(x) \widetilde{\widetilde{C}} I_{1}^{*}(x)$ and $J_{2}^{*}(x) \tilde{\subseteq} J_{1}^{*}(x)$ for all $x \in A_{2}$. As $\mathscr{G}_{1}^{*}$ is a T2ST of $\mathscr{G}, T 1 S S$ corresponding to $T_{1}^{*}(x)$ is a $T 1 S T$ of $\mathscr{G}$ for all $x \in A_{1}$. Also, $\mathscr{G}_{2}^{*}$ is a T2ST of $\mathscr{G}$, T1SS corresponding to $T_{2}^{*}(x)$ is a T1ST of $\mathscr{G}$ for all $x \in A_{2}$. This implies that T1ST corresponding to $T_{2}^{*}(x)$ is a type 1 soft 


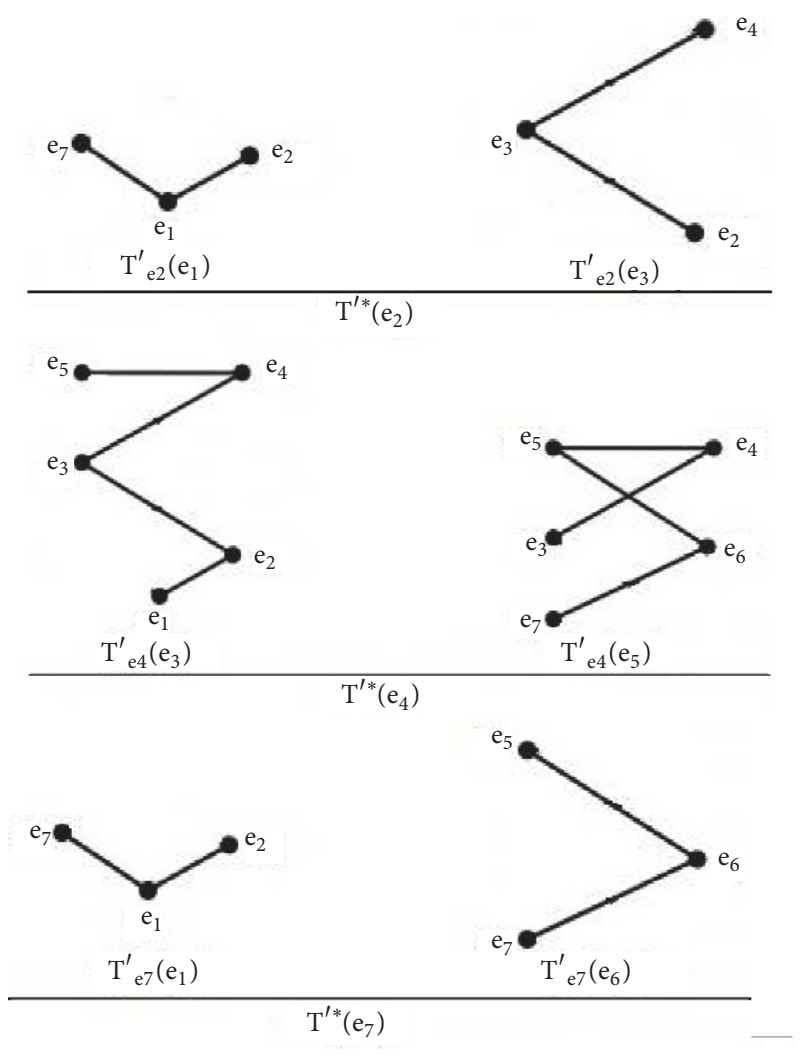

Figure 16: $\mathscr{G}^{\prime *}=\left(T^{\prime *}\left(e_{2}\right), T^{\prime *}\left(e_{4}\right), T^{\prime *}\left(e_{7}\right)\right)$.

subtree of $T 1 S T$ corresponding to $T_{1}^{*}(x)$ for all $x \in A_{2}$. Thus, $\mathscr{G}_{2}^{*}$ is a type 2 soft subtree of $\mathscr{G}_{1}^{*}$.

Definition 42. Let $\mathscr{G}_{1}^{*}=\left\langle I_{1}^{*}, J_{1}^{*}, A_{1}\right\rangle$ and $\mathscr{G}_{2}^{*}=\left\langle I_{2}^{*}, J_{2}^{*}, A_{2}\right\rangle$ be two T2STs of $\mathscr{G}$. The union of $\mathscr{G}_{1}^{*}$ and $\mathscr{G}_{2}^{*}$, is denoted by $\mathscr{G}_{1}^{*} \sqcup \mathscr{G}_{2}^{*}=\mathscr{G}^{*}=\left\langle I^{*}, J^{*}, C\right\rangle$, where $C=A_{1} \cup A_{2}$ is defined $\forall \alpha \in C$, as

$$
I^{*}(\alpha)= \begin{cases}I_{1}^{*}(\alpha), & \text { if } \alpha \in A_{1}-A_{2} \\ I_{2}^{*}(\alpha), & \text { if } \alpha \in A_{2}-A_{1} \\ I_{1}^{*}(\alpha) \widetilde{\cup} I_{2}^{*}(\alpha), & \text { if } \alpha \in A_{1} \cap A_{2}\end{cases}
$$

where $I_{1}^{*}(\alpha) \widetilde{\cup} I_{2}^{*}(\alpha)$ for all $\alpha \in A_{1} \cap A_{2}$ refers to the usual type 1 soft union between the respective T1ST corresponding to $I_{1}^{*}(\alpha)$ and $I_{2}^{*}(\alpha)$, respectively. And,

$$
J^{*}(\alpha)= \begin{cases}J_{1}^{*}(\alpha), & \text { if } \alpha \in A_{1}-A_{2} \\ J_{2}^{*}(\alpha), & \text { if } \alpha \in A_{2}-A_{1} \\ J_{1}^{*}(\alpha) \tilde{\cup} J_{2}^{*}(\alpha), & \text { if } \alpha \in A_{2} \cap A_{1}\end{cases}
$$

where $J_{1}^{*}(\alpha) \tilde{\cup} J_{2}^{*}(\alpha)$ for all $\alpha \in A_{2} \cap A_{1}$ refers to the usual type 1 soft extended union between the respective T1ST corresponding to $J_{1}^{*}(\alpha)$ and $J_{2}^{*}(\alpha)$, respectively.
It can be written as $\mathscr{G}_{1}^{*} \sqcup \mathscr{G}_{2}^{*}=\left\{T^{*}(\alpha)=\left(I^{*}(\alpha), J^{*}(\alpha)\right) \mid\right.$ $\alpha \in C\}$.

Theorem 43. Let $\mathscr{G}_{1}^{*}=\left\langle I_{1}^{*}, J_{1}^{*}, A_{1}\right\rangle$ and $\mathscr{G}_{2}^{*}=\left\langle I_{2}^{*}, J_{2}^{*}, A_{2}\right\rangle$ be two T2STs of $\mathscr{G}$ with $A_{1} \cap A_{2}=\emptyset$. Then $\mathscr{G}_{1}^{*} \sqcup \mathscr{G}_{2}^{*}$ is a T2ST of $G$.

Proof. The union of $\mathscr{G}_{1}^{*}=\left\langle I_{1}^{*}, J_{1}^{*}, A_{1}\right\rangle$ and $\mathscr{G}_{2}^{*}=\left\langle I_{2}^{*}, J_{2}^{*}, A_{2}\right\rangle$ is defined as $\mathscr{G}_{1}^{*} \sqcup \mathscr{G}_{2}^{*}=\mathscr{G}^{*}=\left\langle I^{*}, J^{*}, C\right\rangle$ where $C=A_{1} \cup A_{2}$ for all $\alpha \in C$,

$$
I^{*}(\alpha)= \begin{cases}I_{1}^{*}(\alpha), & \text { if } \alpha \in A_{1}-A_{2} \\ I_{2}^{*}(\alpha), & \text { if } \alpha \in A_{2}-A_{1} \\ I_{1}^{*}(\alpha) \tilde{\cup} I_{2}^{*}(\alpha), & \text { if } \alpha \in A_{1} \cap A_{2}\end{cases}
$$

where $I_{1}^{*}(\alpha) \tilde{\cup} I_{2}^{*}(\alpha)$ for all $\alpha \in A_{1} \cap A_{2}$ refers to the usual type 1 soft union between the respective T1SS corresponding to $I_{1}^{*}(\alpha)$ and $I_{2}^{*}(\alpha)$, respectively. And,

$$
J^{*}(\alpha)= \begin{cases}J_{1}^{*}(\alpha), & \text { if } \alpha \in A_{1}-A_{2} \\ J_{2}^{*}(\alpha), & \text { if } \alpha \in A_{2}-A_{1} \\ J_{1}^{*}(\alpha) \widetilde{\cup} J_{2}^{*}(\alpha), & \text { if } \alpha \in A_{1} \cap A_{2}\end{cases}
$$

where $J_{1}^{*}(\alpha) \widetilde{\cup} J_{2}^{*}(\alpha)$ for all $\alpha \in A_{1} \cap A_{2}$ refers to the usual type 1 soft extended union between the respective T1SS corresponding to $J_{1}^{*}(\alpha)$ and $J_{2}^{*}(\alpha)$, respectively.

Since $\mathscr{G}_{1}^{*}$ is a T2ST of G. Then T1ST corresponding to $\left(I_{1}^{*}(x), J_{1}^{*}(x)\right)$ is a $T 1 S T$ of $\mathscr{G}$ for all $x \in A_{1}-A_{2}$.

Since $\mathscr{G}_{2}^{*}$ is a T2ST of $G$. Then T1ST corresponding to $\left(I_{2}^{*}(x), J_{2}^{*}(x)\right)$ is a $T 1 S T$ of $\mathscr{G}$ for all $x \in A_{2}-A_{1}$.

It is given that $A_{1} \cap A_{2}=\emptyset$. Thus, $\mathscr{G}_{1}^{*} \sqcup \mathscr{G}_{2}^{*}=\mathscr{G}^{*}=$ $\left\langle I^{*}, J^{*}, A_{1} \cup A_{2}\right\rangle$ is a T2ST of $\mathscr{G}$.

If $A_{1} \cap A_{2} \neq \emptyset$ then union of two T2ST may not be a T2ST as it can be seen in the following example.

Example 44. Consider a graph $\mathscr{G}$ defined in Example 40. Let $A=\left\{e_{2}, e_{4}\right\}, B=\left\{e_{2}, e_{7}\right\}$. It may be written that $\mathcal{N} \mathscr{B}_{e_{2}}=$ $\left\{e_{3}, e_{1}\right\}, \mathcal{N} \mathscr{B}_{e_{4}}=\left\{e_{3}, e_{5}\right\}$ and $\mathcal{N} \mathscr{B}_{e_{7}}=\left\{e_{1}, e_{6}\right\}$.

Let $\left[I^{\circ *}, A\right]$ and $\left[J^{\circ *}, A\right]$ be two T2SSs over $X$ and $\mathscr{E}$, respectively, such that $I^{\circ *}(x)=\left(I_{x}^{\circ}, \mathcal{N} \mathscr{B}_{x}\right)$ and $J^{\circ *}(x)=$ $\left(J_{x}^{\circ}, \mathcal{N} \mathscr{B}_{x}\right)$ for all $x \in A$. Define $I_{e_{2}}^{\circ}(z)=\{y \in X \mid z \mathscr{R} y \Longleftrightarrow$ $d(z, y) \leq 1\}, J_{e_{2}}^{\circ}(z)=\left\{\alpha \beta \in \mathscr{E}^{2} \mid\{\alpha, \beta\} \subseteq I_{e_{2}}^{\circ}(z)\right\}$ for all $z \in \mathcal{N} \mathscr{B}_{e_{2}} \subseteq X$ and $I_{e_{4}}^{\circ}(z)=\{y \in X \mid z \mathscr{R} y \Longleftrightarrow d(z, y) \leq 1\}$, $J_{e_{4}}^{\circ}(z)=\left\{\alpha \beta \in \mathscr{E} \mid\{\alpha, \beta\} \subseteq I_{e_{4}}^{\circ}(z)\right\}$ for all $z \in \mathcal{N} \mathscr{B}_{e_{4}} \subseteq X$. Then T2SSs $\left[I^{\circ *}, A\right]$ and $\left[J^{\circ *}, A\right]$ are as follows:

$$
\begin{aligned}
& I^{\circ *}\left(e_{2}\right)=\left\{\left\{e_{1},\left\{e_{1}, e_{2}, e_{7}\right\}\right\},\left\{e_{3},\left\{e_{3}, e_{2}, e_{4}\right\}\right\}\right\}, \\
& J^{\circ *}\left(e_{2}\right)=\left\{\left\{e_{1},\left\{e_{1} e_{2}, e_{7} e_{1}\right\}\right\},\left\{e_{3},\left\{e_{2} e_{3}, e_{3} e_{4}\right\}\right\}\right\}, \\
& I^{\circ *}\left(e_{4}\right)=\left\{\left\{e_{3},\left\{e_{4}, e_{2}, e_{3}\right\}\right\},\left\{e_{5},\left\{e_{6}, e_{5}, e_{4}\right\}\right\}\right\}, \\
& J^{\circ *}\left(e_{4}\right)=\left\{\left\{e_{3},\left\{e_{3} e_{4}, e_{3} e_{2}\right\}\right\},\left\{e_{5},\left\{e_{6} e_{5}, e_{5} e_{4}\right\}\right\}\right\} .
\end{aligned}
$$

Then $\mathscr{G}^{*}=\left(T^{\circ *}\left(e_{2}\right), T^{\circ *}\left(e_{4}\right)\right)$ is a $T 2 S T$ of $\mathscr{G}$.

Let $\left[I^{\prime *}, B\right]$ and $\left[J^{\prime *}, B\right]$ be two T2SSs over $X$ and $\mathscr{E}$, respectively, such that $I^{\prime *}(x)=\left(I_{x}^{\prime}, \mathcal{N} \mathscr{B}_{x}\right)$ and $J^{\prime *}(x)=$ 


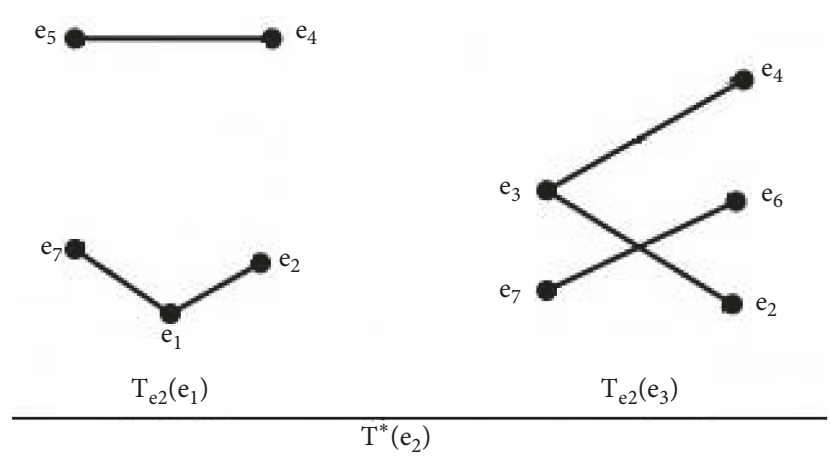

FIGURE 17: $T^{*}\left(e_{2}\right)$.

$\left(J_{x}^{\prime}, \mathcal{N} \mathscr{B}_{x}\right)$ for all $x \in B$. Define $I_{e_{2}}^{\prime}(z)=\{y \in X \mid z \mathscr{R} y \Longleftrightarrow$ $d(z, y) \geq 2\}, J_{e_{1}}^{\prime}(z)=\left\{\alpha \beta \in \mathscr{E} \mid\{\alpha, \beta\} \subset I_{e_{2}}^{\prime}(z)\right\}$ for all $z \in \mathcal{N} \mathscr{B}_{e_{2}} \subseteq X$ and $I_{e_{7}}^{\prime}(z)=\{y \in X \mid z \mathscr{R} y \Longleftrightarrow d(z, y) \geq 1\}$, $J_{e_{7}}^{\prime}(z)=\left\{\alpha \beta \in \mathscr{E} \mid\{\alpha, \beta\} \subset I_{e_{7}}^{\prime}(z)\right\}$ for all $z \in \mathscr{N}_{e_{e_{7}}} \subseteq X$. Then T2SSs $\left[I^{\prime *}, B\right]$ and $\left[J^{\prime *}, B\right]$ are as follows:

$$
\begin{aligned}
& I^{\prime *}\left(e_{2}\right)=\left\{\left\{e_{1},\left\{e_{4}, e_{5}\right\}\right\},\left\{e_{3},\left\{e_{6}, e_{7}\right\}\right\},\right. \\
& J^{\prime *}\left(e_{2}\right)=\left\{\left\{e_{1},\left\{e_{4} e_{5}\right\}\right\},\left\{e_{3},\left\{e_{6} e_{7}\right\}\right\}\right\}, \\
& I^{\prime *}\left(e_{7}\right)=\left\{\left\{e_{1},\left\{e_{3}, e_{4}, e_{5}, e_{6}\right\}\right\},\left\{e_{6},\left\{e_{1}, e_{2}, e_{3}, e_{4}\right\}\right\}\right\}, \\
& J^{\prime *}\left(e_{7}\right) \\
& \quad=\left\{\left\{e_{1},\left\{e_{3} e_{4}, e_{4} e_{5}, e_{5} e_{6}\right\}\right\},\left\{e_{6},\left\{e_{1} e_{2}, e_{2} e_{3}, e_{3} e_{4}\right\}\right\}\right\} .
\end{aligned}
$$

Then $\mathscr{G}^{\prime *}=\left(T^{\prime *}\left(e_{2}\right), T^{\prime *}\left(e_{4}\right), T^{\prime *}\left(e_{7}\right)\right)$ is a T2ST of $\mathscr{G}$. By the definition of union of T2STs,

$$
I^{*}\left(e_{4}\right)=I^{\circ *}\left(e_{4}\right)
$$

and $J^{*}\left(e_{4}\right)=J^{\circ *}\left(e_{4}\right)$,

$$
e_{4} \in A-B
$$

$$
I^{*}\left(e_{7}\right)=I^{\prime *}\left(e_{7}\right)
$$

and $J^{*}\left(e_{7}\right)=J^{*}\left(e_{7}\right)$,

$$
\begin{aligned}
& e_{7} \in B-A, \\
& \text { and } I^{*}\left(e_{2}\right)=I^{* *}\left(e_{2}\right) \cup I^{\prime *}\left(e_{2}\right) \\
& J^{\circ *}\left(e_{2}\right) \widetilde{\cup} J^{\prime *}\left(e_{2}\right), \\
& e_{2} \in B \cap A .
\end{aligned}
$$

By routine calculations, it is easy to see that T1SGs corresponding to $T^{*}\left(e_{4}\right)=T^{\circ *}\left(e_{4}\right)$ and $T^{*}\left(e_{7}\right)=T^{\prime *}\left(e_{7}\right)$ are T1STs. But T1SG corresponding to $T^{*}\left(e_{2}\right)=T^{\circ *}\left(e_{2}\right) \widetilde{\cup} T^{\prime *}\left(e_{2}\right)$ is a disconnected type 2 soft forest, as shown in Figure 17. Therefore, $\mathscr{G}_{1}^{*} \sqcup \mathscr{G}_{2}^{*}=\mathscr{G}^{*}=\left\langle I^{*}, J^{*}, A \cup B\right\rangle$ is not a $T 2 S T$ of $\mathscr{G}$.

Lemma 45. Let $\mathscr{G}_{1}^{*}=\left\langle S_{1}^{*}, T_{1}^{*}, A_{1}\right\rangle$ and $\mathscr{G}_{2}^{*}=\left\langle S_{2}^{*}, T_{2}^{*}, A_{2}\right\rangle$ be two T2STs of $\mathscr{G}$. If $A_{1} \cap A_{2}=\emptyset$, then their union is a T2ST of $\mathscr{G}$.

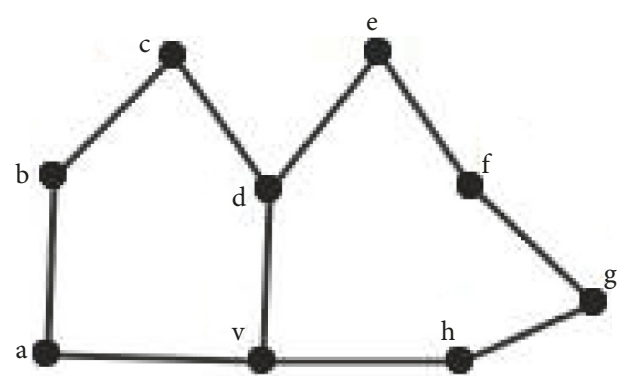

FIGURE 18: Simple graph.

Definition 46. Let $\mathscr{G}_{1}^{*}=\left\langle I_{1}^{*}, J_{1}^{*}, A_{1}\right\rangle$ and $\mathscr{G}_{2}^{*}=\left\langle I_{2}^{*}, J_{2}^{*}, A_{2}\right\rangle$ be two T2STs of $\mathscr{G}$. The intersection of $\mathscr{G}_{1}^{*}$ and $\mathscr{G}_{2}^{*}$, denoted by $\mathscr{G}_{1}^{*} \sqcap \mathscr{G}_{2}^{*}=\mathscr{G}^{*}=\left\langle I^{*}, J^{*}, C\right\rangle$, where $C=A \cap B$ is defined as $I^{*}(\alpha)=I_{1}^{*}(\alpha) \widetilde{\cap} I_{2}^{*}(\alpha)$, for all $\alpha \in A \cap B$, where $I_{1}^{*}(\alpha) \widetilde{\cap} I_{2}^{*}(\alpha)$ for all $\alpha \in A \cap B$ refers to the usual type 1 soft intersection between the respective T1SS corresponding to $I_{1}^{*}(\alpha)$ and $I_{2}^{*}(\alpha)$, respectively. And $J^{*}(\alpha)=J_{1}^{*}(\alpha) \widetilde{\cap} J_{2}^{*}(\alpha)$ for all $\alpha \in A \cap B$ where $J_{1}^{*}(\alpha) \widetilde{n} J_{2}^{*}(\alpha)$ for all $\alpha \in A \cap B$ refers to the usual type 1 soft intersection between the respective T1SS corresponding to $J_{1}^{*}(\alpha)$ and $J_{2}^{*}(\alpha)$, respectively.

It can be written as $\mathscr{G}_{1}^{*} \sqcap \mathscr{G}_{2}^{*}=\left\{T^{*}(\alpha)=\left(I^{*}(\alpha), J^{*}(\alpha)\right) \mid\right.$ $\alpha \in C\}$.

The intersection of two T2STs may not be T2ST as it can be seen in the following example.

Example 47. Consider a simple graph $\mathscr{G}$ shown in Figure 18. Let $A=\{a, h\}$ and $B=\{b, h\}$. It may be written that $\mathscr{N} \mathscr{B}_{a}=$ $\{v, b\}, \mathcal{N} \mathscr{B}_{h}=\{v, g\}$ and $\mathscr{N} \mathscr{B}_{b}=\{a, c\}$.

Let $\left[I^{\circ *}, A\right]$ and $\left[J^{\circ *}, A\right]$ be two T2SSs over $X$ and $\mathscr{E}$, respectively, such that $I^{\circ *}(x)=\left(I_{x}^{\circ}, \mathscr{N}_{x}\right)$ and $J^{\circ *}(x)=$ $\left(J_{x}^{\circ}, \mathscr{N}_{x}\right)$ for all $x \in A$. Define $I_{a}^{\circ}(z)=\{y \in X \mid z \mathscr{R} y \Longleftrightarrow$ $d(z, y) \leq 1\}, J_{a}^{\circ}(z)=\left\{\alpha \beta \in \mathscr{E} \mid\{\alpha, \beta\} \subset I_{a}^{\circ}(z)\right\}$ for all $z \in \mathscr{N} \mathscr{B}_{a} \subseteq X$ and $I_{h}^{\circ}(z)=\{y \in X \mid z \mathscr{R} y \Longleftrightarrow d(z, y) \leq 1\}$, $J_{h}^{\circ}(z)=\left\{\alpha \beta \in \mathscr{E} \mid\{\alpha, \beta\} \subset I_{h}^{\circ}(z)\right\}$ for all $z \in \mathscr{N}_{\mathscr{B}_{h}} \subseteq X$. Then T2SSs $\left[I^{\circ *}, A\right]$ and $\left[J^{\circ *}, A\right]$ are as follows:

$$
\begin{aligned}
& I^{* *}(a)=\{\{b,\{a, b, c\}\},\{v,\{b, v, h, d\}\}\}, \\
& J^{\circ *}(a)=\{\{b,\{a b, b c\}\},\{v,\{v b, v h, v d\}\}\}, \\
& I^{\circ *}(h)=\{\{v,\{a, d, h, v\}\},\{g,\{g, f, h\}\}\}, \\
& \left.J^{\circ *}(h)=\{v,\{v d, v a, v h\}\},\{g,\{g f, g h\}\}\right\} .
\end{aligned}
$$

Then $\mathscr{G}^{*}=\left(T^{\circ *}(a), T^{\circ *}(g)\right)$ is a $T 2 S T$ of $\mathscr{G}$.

Let $\left[I^{\prime *}, B\right]$ and $\left[J^{\prime *}, B\right]$ be two T2SSs over $X$ and $\mathscr{E}$, respectively, such that $I^{\prime *}(x)=\left(I_{x}^{\prime}, \mathcal{N}_{\mathscr{B}_{x}}\right)$ and $J^{\prime *}(x)=$ $\left(J_{x}^{\prime}, \mathcal{N}_{x}\right)$ for all $x \in B$. Define $I_{b}^{\prime}(z)=\{y \in X \mid z \mathscr{R} y \Longleftrightarrow$ $d(z, y) \geq 3\}, J_{b}^{\prime}(z)=\left\{\alpha \beta \in \mathscr{E} \mid\{\alpha, \beta\} \in I_{b}^{\prime}(z)\right\}$ for all $z \in$ $\mathcal{N} \mathscr{B}_{b} \subseteq X$ and $I_{h}^{\prime}(z)=\{y \in X \mid z \mathscr{R} y \Longleftrightarrow 1 \leq d(z, y) \leq 3\}$, $J_{h}^{\prime}(z)=\left\{\alpha \beta \in \mathscr{E} \mid\{\alpha, \beta\} \in I_{h}^{\prime}(z)\right\}$ for all $z \in \mathscr{N}_{\mathscr{B}_{h}} \subseteq X$. Then T2SSs $\left[I^{\prime *}, B\right]$ and $\left[J^{\prime *}, B\right]$ are as follows: 


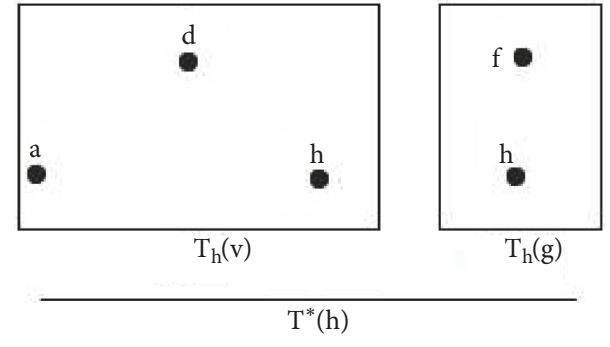

Figure 19: $\mathscr{G}_{1}^{*} \sqcap \mathscr{G}_{2}^{*}=\mathscr{G}^{*}=\left\langle I^{*}, J^{*}, A \cap B\right\rangle$.

$I^{\prime *}(b)=\{\{a,\{e, f, g\}\},\{c,\{f, g, h\}\}\}$,
$J^{\prime *}(b)=\{\{a,\{e f, f g\}\},\{c,\{f g, g h\}\}$,

$I^{\prime *}(h)$

$=\{\{v,\{e, f, g, d, a, b, h, c\}\},\{g,\{d, e, f, h, v, a\}\}$,

$J^{\prime *}(h)$

$=\{\{v,\{e f, f g, e d, v d, d c\}\},\{g,\{e f, e d, v h, v a, v d\}\}\}$.

Then $\mathscr{G}^{\prime *}=\left(T^{\prime *}(b), T^{\prime *}(h)\right)$ is a $T 2 S T$ of $\mathscr{G}$. By the definition of intersection of T2ST,

$$
\begin{aligned}
I^{*}(h) & =I^{* *}(h) \widetilde{\cap} I^{\prime *}(h) \\
\text { and } J^{*}(h) & =J^{* *}(h) \widetilde{\cap} J^{\prime *}(h), \\
\{h\} & =B \cap A .
\end{aligned}
$$

By routine calculations, it is easy to see that T1SG corresponding to $T^{*}(h)=T^{* *}(h) \widetilde{\cap} T^{\prime *}(h)$ is a disconnected $T 1 S G$, as shown in Figure 19. Therefore, $\mathscr{G}_{1}^{*} \sqcap \mathscr{G}_{2}^{*}=\mathscr{G}^{*}=\left\langle I^{*}, J^{*}, A \cup\right.$ $B\rangle$ is not a $T 2 S T$ of $\mathscr{G}$.

Definition 48. Let $\mathscr{G}_{1}^{*}=\left\langle I_{1}^{*}, J_{1}^{*}, A_{1}\right\rangle$ and $\mathscr{G}_{2}^{*}=\left\langle I_{2}^{*}, J_{2}^{*}, A_{2}\right\rangle$

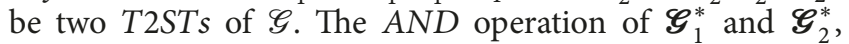
denoted by $\mathscr{G}_{1}^{*} \bigwedge \mathscr{G}_{2}^{*}=\mathscr{G}^{*}=\left\langle I^{*}, J^{*}, A \times B\right\rangle$ defined by $I^{*}(\gamma, \eta)=I_{1}^{*}(\gamma) \widetilde{\wedge} I_{2}^{*}(\eta), J^{*}(\gamma, \eta)=J_{1}^{*}(\gamma) \widetilde{\wedge} J_{2}^{*}(\eta)$ for all $(\gamma, \eta) \epsilon$ $A \times B$, where $I_{1}^{*}(\gamma) \widetilde{\wedge} I_{2}^{*}(\eta)$ for all $(\gamma, \eta) \in A \times B$ refers to the usual type 1 soft $A N D$ operation between the respective T1SS corresponding to $I_{1}^{*}(\gamma)$ and $I_{2}^{*}(\eta)$ respectively and $J_{1}^{*}(\gamma) \tilde{\wedge} J_{2}^{*}(\eta)$ for all $(\gamma, \eta) \in A \times B$ refers to the usual type 1 soft $A N D$ operation between the respective T1SS corresponding to $J_{1}^{*}(\gamma)$ and $J_{2}^{*}(\eta)$ respectively.

Example 49. Consider a simple graph $\mathscr{G}=(X, \mathscr{E})$, where $X=\left\{e_{1}, e_{2}, e_{3}, e_{4}, e_{5}, e_{6}, e_{7}, e_{8}\right\}$ and $\mathscr{E}=\left\{e_{1} e_{2}, e_{2} e_{3}, e_{3} e_{4}, e_{5} e_{4}\right.$, $\left.e_{5} e_{6}, e_{6} e_{7}, e_{7} e_{8}, e_{8} e_{1}\right\}$. Let $A=\left\{e_{1}, e_{8}\right\}, B=\left\{e_{5}\right\}$. It may be written that $\mathcal{N} \mathscr{B}_{e_{1}}=\left\{e_{8}, e_{2}\right\}, \mathcal{N} \mathscr{B}_{e_{8}}=\left\{e_{7}, e_{1}\right\}$ and $\mathcal{N} \mathscr{B}_{e_{5}}=$ $\left\{e_{4}, e_{6}\right\}$.

Let $\left[I^{\circ *}, A\right]$ and $\left[J^{\circ *}, A\right]$ be two T2SSs over $X$ and $\mathscr{E}$, respectively, such that $I^{\circ *}(x)=\left(I_{x}^{\circ}, \mathcal{N} \mathscr{B}_{x}\right)$ and $J^{\circ *}(x)=$ $\left(J_{x}^{\circ}, \mathcal{N} \mathscr{B}_{x}\right)$ for all $x \in A$. Define $I_{e_{1}}^{\circ}(z)=\{y \in X \mid z \mathscr{R} y \Longleftrightarrow$ $d(z, y) \leq 1\}, J_{e_{1}}^{\circ}(z)=\left\{\alpha \beta \in \mathscr{E} \mid\{\alpha, \beta\} \subset I_{e_{1}}^{\circ}(z)\right\}$ for all $z \in \mathcal{N} \mathscr{B}_{e_{1}} \subseteq X$ and $I_{e_{8}}^{\circ}(z)=\{y \in X \mid z \mathscr{R} y \Longleftrightarrow d(z, y) \leq 1\}$,
$J_{e_{8}}^{\circ}(z)=\left\{\alpha \beta \in \mathscr{E} \mid\{\alpha, \beta\} \subset I_{e_{8}}^{\circ}(z)\right\}$ for all $z \in \mathcal{N} \mathscr{B}_{e_{8}} \subseteq X$. Then T2SSs $\left[I^{\circ *}, A\right]$ and $\left[J^{\circ *}, A\right]$ are as follows:

$$
\begin{aligned}
& I^{\circ *}\left(e_{1}\right)=\left\{\left\{e_{2},\left\{e_{1}, e_{2}, e_{3}\right\}\right\},\left\{e_{8},\left\{e_{1}, e_{8}, e_{7}\right\}\right\}\right\}, \\
& J^{\circ *}\left(e_{1}\right)=\left\{\left\{e_{2},\left\{e_{1} e_{2}, e_{2} e_{3}\right\}\right\},\left\{e_{8},\left\{e_{1} e_{8}, e_{8} e_{7}\right\}\right\}\right\}, \\
& I^{\circ *}\left(e_{8}\right)=\left\{\left\{e_{1},\left\{e_{8}, e_{1}, e_{7}\right\}\right\},\left\{e_{7},\left\{e_{6}, e_{7}, e_{8}\right\}\right\}\right\}, \\
& J^{\circ *}\left(e_{8}\right)=\left\{\left\{e_{1},\left\{e_{2} e_{1}, e_{1} e_{8}, e_{8} e_{7}, e_{7} e_{6}\right\}\right\},\right. \\
& \left.\quad\left\{e_{7},\left\{e_{1} e_{8}, e_{7} e_{8}, e_{6} e_{7}, e_{6} e_{5}\right\}\right\}\right\} .
\end{aligned}
$$

Then $\mathscr{G}^{*}=\left(T^{\circ *}\left(e_{1}\right), T^{\circ *}\left(e_{8}\right)\right)$ is a $T 2 S T$ of $\mathscr{G}$.

Let $\left[I^{\prime *}, B\right]$ and $\left[J^{\prime *}, B\right]$ be two T2SSs over $X$ and $\mathscr{E}$, respectively, such that $I^{\prime *}(x)=\left(J_{x}^{\prime}, \mathscr{N}_{x}\right)$ and $J^{\prime *}(x)=$ $\left(J_{x}^{\prime}, \mathcal{N} \mathscr{B}_{x}\right)$ for all $x \in B$. Define $I_{e_{5}}^{\prime}(z)=\{y \in X \mid z \mathscr{R} y \Longleftrightarrow$ $d(z, y) \geq 1\}, J_{e_{5}}^{\prime}(z)=\left\{\alpha \beta \in \mathscr{E} \mid\{\alpha, \beta\} \subset I_{e_{5}}^{\prime}(z)\right\}$ for all $z \in \mathcal{N} \mathscr{B}_{e_{5}} \subseteq X$. Then T2SSs $\left[I^{\prime *}, B\right]$ and $\left[J^{\prime *}, B\right]$ are as follows:

$$
\begin{aligned}
& I^{\prime *}\left(e_{5}\right)=\left\{\left\{e_{6},\left\{e_{4}, e_{2}, e_{3}, e_{1}, e_{5}, e_{8}, e_{7}\right\}\right\},\right. \\
& \left.\left\{e_{4},\left\{e_{6}, e_{5}, e_{1}, e_{2}, e_{3}, e_{8}, e_{7}\right\}\right\}\right\}, \\
& J^{\prime *}\left(e_{5}\right)=\left\{\left\{e_{6},\left\{e_{1} e_{2}, e_{2} e_{3}, e_{3} e_{4}, e_{5} e_{4}, e_{1} e_{8}, e_{7} e_{8}\right\}\right\},\right. \\
& \left.\left\{e_{4},\left\{e_{3} e_{2}, e_{2} e_{1}, e_{7} e_{6}, e_{6} e_{5}, e_{5} e_{4}, e_{1} e_{8}, e_{5} e_{4}, e_{7} e_{8}\right\}\right\}\right\},
\end{aligned}
$$

Then $\mathscr{G}^{\prime *}=\left(T^{\prime *}\left(e_{5}\right)\right)$ is a T2ST of $\mathscr{G}$. The AND operation on $\mathscr{G}^{*}$ and $\mathscr{G}^{\prime *}$ is defined as in the following:

$$
\begin{aligned}
& I^{*}\left(e_{1}, e_{5}\right)=I^{\circ *}\left(e_{1}\right) \tilde{\wedge} I^{\prime *}\left(e_{5}\right) \\
& =\left\{\left\{\left(e_{2}, e_{6}\right),\left\{e_{2}, e_{3}, e_{1}\right\}\right\},\left\{\left(e_{2}, e_{4}\right),\left\{e_{1}, e_{3}, e_{2}\right\}\right\},\right. \\
& \left.\quad\left\{\left(e_{8}, e_{6}\right),\left\{e_{1}, e_{8}, e_{7}\right\}\right\},\left\{\left(e_{8}, e_{4}\right),\left\{e_{1}, e_{8}, e_{7}\right\}\right\}\right\}, \\
& J^{*}\left(e_{1}, e_{5}\right)=J^{\circ *}\left(e_{1}\right) \tilde{\wedge} J^{\prime *}\left(e_{5}\right) \\
& =\left\{\left\{\left(e_{2}, e_{6}\right),\left\{e_{2} e_{3}, e_{2} e_{1}\right\}\right\},\left\{\left(e_{2}, e_{4}\right),\left\{e_{1} e_{2}, e_{3} e_{2}\right\}\right\},\right. \\
& \left.\quad\left\{\left(e_{8}, e_{6}\right),\left\{e_{1} e_{8}, e_{8} e_{7}\right\}\right\},\left\{\left(e_{8}, e_{4}\right),\left\{e_{1} e_{8}, e_{8} e_{7}\right\}\right\}\right\}, \\
& I^{*}\left(e_{8}, e_{5}\right)=I^{\circ *}\left(e_{8}\right) \tilde{\wedge} I^{\prime *}\left(e_{5}\right) \\
& =\left\{\left\{\left(e_{1}, e_{6}\right),\left\{e_{7}, e_{8}, e_{1}\right\}\right\},\left\{\left(e_{1}, e_{4}\right),\left\{e_{1}, e_{8}, e_{7}\right\}\right\},\right. \\
& \left.\quad\left\{\left(e_{7}, e_{6}\right),\left\{e_{8}, e_{7}\right\}\right\},\left\{\left(e_{7}, e_{4}\right),\left\{e_{6}, e_{7}, e_{8}\right\}\right\}\right\}, \\
& J^{*}\left(e_{8}, e_{5}\right)=J^{\circ *}\left(e_{8}\right) \tilde{\wedge} J^{\prime *}\left(e_{5}\right) \\
& \quad=\left\{\left\{\left(e_{1}, e_{6}\right),\left\{e_{7} e_{8}, e_{1} e_{8}\right\}\right\},\left\{\left(e_{1}, e_{4}\right),\left\{e_{1} e_{8}, e_{7} e_{8}\right\}\right\},\right. \\
& \left.\quad\left\{\left(e_{7}, e_{6}\right),\left\{e_{8} e_{7}\right\}\right\},\left\{\left(e_{7}, e_{4}\right),\left\{e_{6} e_{7}, e_{8} e_{7}\right\}\right\}\right\} .
\end{aligned}
$$

The AND operation on $\mathscr{G}^{*}$ and $\mathscr{G}^{\prime *}$ is shown in Figure 20.

Definition 50. Let $\mathscr{G}_{1}^{*}=\left\langle I_{1}^{*}, J_{1}^{*}, A_{1}\right\rangle$ and $\mathscr{G}_{2}^{*}=\left\langle I_{2}^{*}, J_{2}^{*}, A_{2}\right\rangle$ be two T2STs of $\mathscr{G}$. The OR operation of $\mathscr{G}_{1}^{*}$ and $\mathscr{G}_{2}^{*}$, denoted by $\mathscr{G}_{1}^{*} \bigvee \mathscr{G}_{2}^{*}=\mathscr{G}^{*}=\left\langle I^{*}, J^{*}, A \times B\right\rangle$ defined by $I^{*}(\gamma, \eta)=I_{1}^{*}(\gamma) \widetilde{V} I_{2}^{*}(\eta), J^{*}(\gamma, \eta)=J_{1}^{*}(\gamma) \widetilde{V} J_{2}^{*}(\eta)$ for all $(\gamma, \eta) \in$ 


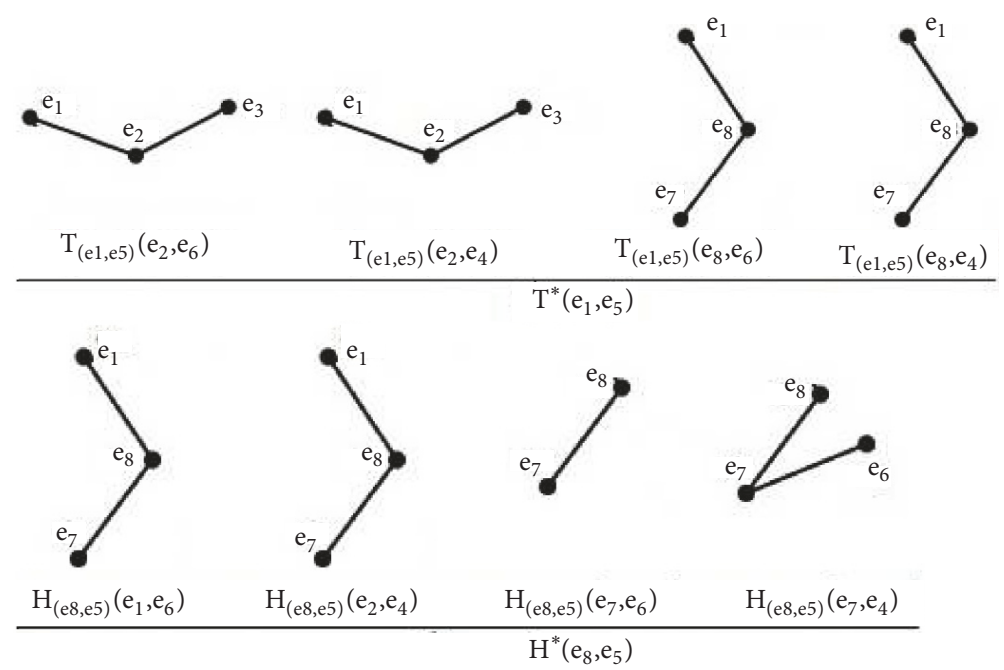

FIGURE 20: $A N D$ operation.

$A \times B$, where $I_{1}^{*}(\gamma) \widetilde{V} I_{2}^{*}(\eta)$ for all $(\gamma, \eta) \in A \times B$ refers to the usual type 1 soft $O R$ operation between the respective T1SS corresponding to $I_{1}^{*}(\gamma)$ and $I_{2}^{*}(\eta)$ respectively and $J_{1}^{*}(\gamma) \widetilde{V} J_{2}^{*}(\eta)$ for all $(\gamma, \eta) \in A \times B$ refers to the usual type 1 soft $O R$ operation between the respective T1SS corresponding to $J_{1}^{*}(\gamma)$ and $J_{2}^{*}(\eta)$ respectively.

Example 51. Consider a simple graph $\mathscr{G}=(X, \mathscr{E})$ defined in Example 49. Let $A=\left\{e_{3}, e_{5}\right\}, B=\left\{e_{6}\right\}$. It may be written that $\mathcal{N} \mathscr{B}_{e_{3}}=\left\{e_{4}, e_{2}\right\}, \mathscr{N}_{e_{6}}=\left\{e_{7}, e_{5}\right\}$ and $\mathscr{N} \mathscr{B}_{e_{5}}=\left\{e_{4}, e_{6}\right\}$.

Let $\left[I^{\circ *}, A\right]$ and $\left[J^{\circ *}, A\right]$ be two T2SSs over $X$ and $\mathscr{E}$ respectively, such that $I^{\circ *}(x)=\left(I_{x}^{\circ}, \mathscr{N} \mathscr{B}_{x}\right)$ and $J^{\circ *}(x)=$ $\left(J_{x}^{\circ}, \mathcal{N} \mathscr{B}_{x}\right)$ for all $x \in A$. Define $I_{e_{3}}^{\circ}(z)=\{y \in X \mid z \mathscr{R} y \Longleftrightarrow$ $d(z, y) \leq 1\}, J_{e_{3}}^{\circ}(z)=\left\{\alpha \beta \in \mathscr{E} \mid\{\alpha, \beta\} \subset I_{e_{3}}^{\circ}(z)\right\}$ for all $z \in \mathscr{N}_{e_{3}} \subseteq X, I_{e_{5}}^{\circ}(z)=\{y \in X \mid z \mathscr{R} y \Longleftrightarrow d(z, y) \geq 3\}$, $J_{e_{5}}^{\circ}(z)=\left\{\alpha \beta \in \mathscr{E} \mid\{\alpha, \beta\} \subset I_{e_{5}}^{\circ}(z)\right\}$ for all $z \in \mathscr{N}_{B_{5}} \subseteq X$. Then T2SSs $\left[I^{\circ *}, A\right]$ and $\left[J^{\circ *}, A\right]$ are as follows:

$$
\begin{aligned}
& I^{\circ *}\left(e_{3}\right)=\left\{\left\{e_{2},\left\{e_{1}, e_{2}, e_{3}\right\}\right\},\left\{e_{4},\left\{e_{3}, e_{4}, e_{5}\right\}\right\},\right. \\
& J^{\circ *}\left(e_{3}\right)=\left\{\left\{e_{2},\left\{e_{1} e_{2}, e_{2} e_{3}\right\}\right\},\left\{e_{4},\left\{e_{3} e_{4}, e_{4} e_{5}\right\}\right\}\right\}, \\
& I^{\circ *}\left(e_{5}\right)=\left\{\left\{e_{4},\left\{e_{8}, e_{1}, e_{7}\right\}\right\},\left\{e_{6},\left\{e_{1}, e_{2}, e_{3}\right\}\right\}\right\}, \\
& J^{\circ *}\left(e_{5}\right)=\left\{\left\{e_{4},\left\{e_{8} e_{7}, e_{1} e_{8}\right\}\right\},\left\{e_{6},\left\{e_{1} e_{2}, e_{2} e_{3}\right\}\right\}\right\} .
\end{aligned}
$$

Then $\mathscr{G}^{*}=\left(T^{\circ *}\left(e_{2}\right), T^{\circ *}\left(e_{4}\right)\right)$ is a $T 2 S T$ of $\mathscr{G}$.

Let $\left[I^{\prime *}, B\right]$ and $\left[J^{\prime *}, B\right]$ be two T2SSs over $X$ and $\mathscr{E}$, respectively, such that $I^{\prime *}(x)=\left(I_{x}^{\prime}, \mathscr{N}_{x}\right)$ and $J^{\prime *}(x)=$ $\left(J_{x}^{\prime}, \mathcal{N} \mathscr{B}_{x}\right)$ for all $x \in B$. Define $I_{e_{6}}^{\prime}(z)=\{y \in X \mid z \mathscr{R} y \Longleftrightarrow$ $d(z, y) \leq 1\}, J_{e_{6}}^{\prime}(z)=\left\{\alpha \beta \in \mathscr{E} \mid\{\alpha, \beta\} \subset I_{e_{6}}^{\prime}(z)\right\}$ for all $z \in \mathscr{N}_{e_{6}} \subseteq X$. Then T2SSs $\left[I^{\prime *}, B\right]$ and $\left[J^{\prime *}, B\right]$ are as follows:

$$
\begin{gathered}
I^{\prime *}\left(e_{6}\right)=\left\{\left\{e_{7},\left\{e_{4}, e_{2}, e_{3}, e_{1}, e_{5}\right\}\right\},\right. \\
\left.\left\{e_{5},\left\{e_{1}, e_{2}, e_{3}, e_{8}, e_{7}\right\}\right\}\right\},
\end{gathered}
$$

$$
\begin{gathered}
J^{\prime *}\left(e_{6}\right)=\left\{\left\{e_{7},\left\{e_{1} e_{2}, e_{2} e_{3}, e_{3} e_{4}, e_{5} e_{4}\right\}\right\},\right. \\
\left\{e_{5},\left\{e_{3} e_{2}, e_{2} e_{1}, e_{1} e_{8}, e_{7} e_{8}\right\}\right\},
\end{gathered}
$$

Then $\mathscr{G}^{\prime *}=\left(T^{\prime *}\left(e_{6}\right)\right)$ is a T2ST of $\mathscr{G}$. The OR operation on $\mathscr{G}^{*}$ and $\mathscr{G}^{\prime *}$ is defined as in the following:

$$
\begin{aligned}
I^{*} & \left(e_{3}, e_{6}\right)=I^{\circ *}\left(e_{3}\right) \tilde{\wedge} I^{\prime *}\left(e_{6}\right) \\
& =\left\{\left\{\left(e_{2}, e_{7}\right),\left\{e_{4}, e_{2}, e_{3}, e_{1}, e_{5}\right\}\right\},\right. \\
& \left\{\left(e_{2}, e_{5}\right),\left\{e_{7}, e_{2}, e_{3}, e_{1}, e_{8}\right\}\right\}, \\
& \left\{\left(e_{4}, e_{7}\right),\left\{e_{4}, e_{2}, e_{3}, e_{1}, e_{5}\right\}\right\}, \\
& \left.\left\{\left(e_{4}, e_{5}\right),\left\{e_{4}, e_{2}, e_{3}, e_{1}, e_{5}, e_{7}, e_{8}\right\}\right\}\right\}, \\
J^{*} & \left(e_{3}, e_{6}\right)=J^{\circ *}\left(e_{3}\right) \tilde{\wedge} J^{\prime *}\left(e_{6}\right) \\
& =\left\{\left\{\left(e_{2}, e_{7}\right),\left\{e_{1} e_{2}, e_{2} e_{3}, e_{3} e_{4}, e_{5} e_{4}\right\}\right\},\right. \\
& \left\{\left(e_{2}, e_{5}\right),\left\{e_{1} e_{2}, e_{2} e_{3}, e_{1} e_{8}, e_{7} e_{8}\right\}\right\}, \\
& \left\{\left(e_{4}, e_{7}\right),\left\{e_{1} e_{2}, e_{3} e_{2}, e_{4} e_{3}, e_{4} e_{5}\right\}\right\}, \\
& \left.\left\{\left(e_{4}, e_{5}\right),\left\{e_{1} e_{2}, e_{2} e_{3}, e_{3} e_{4}, e_{5} e_{4}, e_{1} e_{8}, e_{8} e_{7}\right\}\right\}\right\}, \\
I^{*} & \left(e_{5}, e_{6}\right)=I^{\circ *}\left(e_{5}\right) \tilde{\wedge} I^{\prime *}\left(e_{6}\right) \\
& =\left\{\left\{\left(e_{4}, e_{7}\right),\left\{e_{4}, e_{2}, e_{3}, e_{1}, e_{5}, e_{7}, e_{8}\right\}\right\},\right. \\
& \left\{\left(e_{4}, e_{5}\right),\left\{e_{7}, e_{2}, e_{3}, e_{1}, e_{8}\right\}\right\}, \\
& \left\{\left(e_{6}, e_{7}\right),\left\{e_{1}, e_{2}, e_{3}, e_{4}, e_{5}\right\}\right\}, \\
& \left.\left\{\left(e_{6}, e_{5}\right),\left\{e_{1}, e_{2}, e_{3}, e_{8}, e_{7}\right\}\right\}\right\}, \\
J^{*} & \left(e_{8}, e_{5}\right)=J^{\circ *}\left(e_{8}\right) \tilde{\wedge} J^{\prime *}\left(e_{5}\right) \\
& =\left\{\left\{\left(e_{4}, e_{7}\right),\left\{e_{7} e_{8}, e_{1} e_{8}, e_{4} e_{5}, e_{3} e_{4}, e_{3} e_{2}, e_{1} e_{2}\right\}\right\},\right.
\end{aligned}
$$




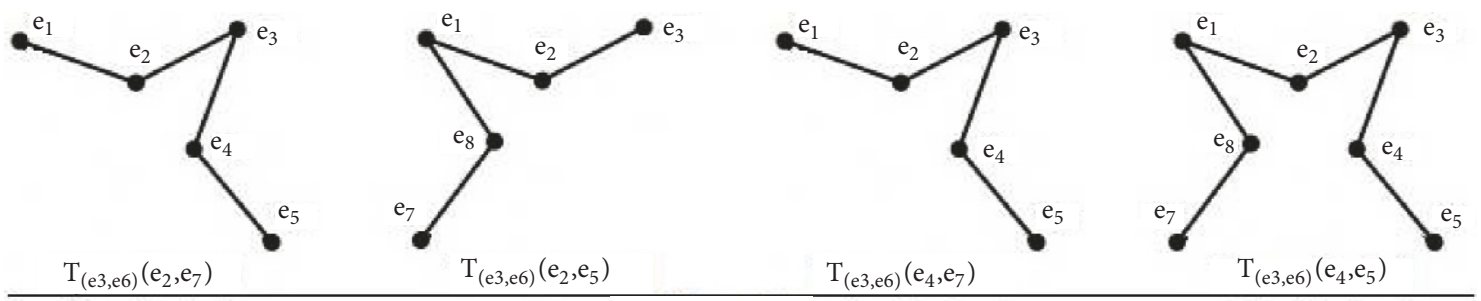

$\mathrm{T}^{*}\left(\mathrm{e}_{3}, \mathrm{e}_{6}\right)$
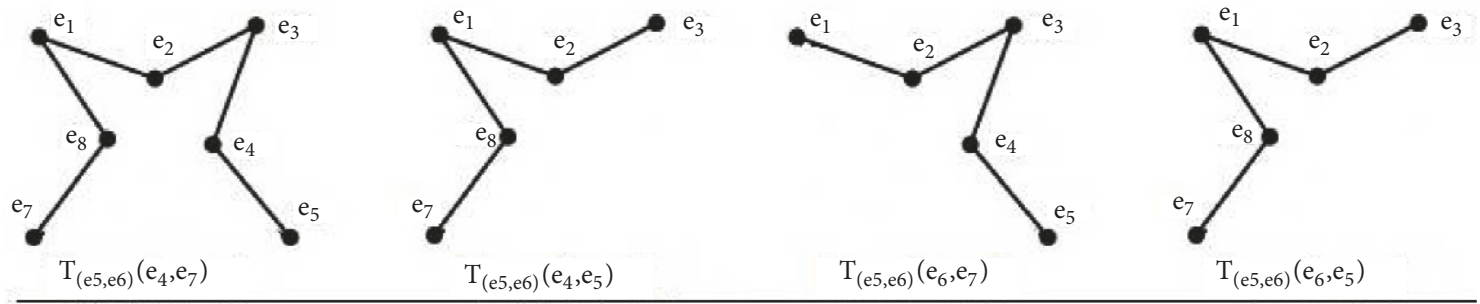

$\mathrm{T}^{*}\left(\mathrm{e}_{5}, \mathrm{e}_{6}\right)$

FIGURE 21: OR operation.

$$
\begin{aligned}
& \left\{\left(e_{4}, e_{5}\right),\left\{e_{1} e_{2}, e_{2} e_{3}, e_{1} e_{8}, e_{8} e_{7}\right\}\right\} \\
& \left\{\left(e_{6}, e_{7}\right),\left\{e_{1} e_{2}, e_{2} e_{3}, e_{3} e_{4}, e_{4} e_{5}\right\}\right\} \\
& \left.\left\{\left(e_{6}, e_{5}\right),\left\{e_{1} e_{2}, e_{2} e_{3}, e_{7} e_{8}, e_{1} e_{8}\right\}\right\}\right\}
\end{aligned}
$$

The $O R$ operation on $\mathscr{G}^{*}$ and $\mathscr{G}^{*}$ is shown in Figure 21.

\section{Conclusion}

In above study, we have characterized type 2 soft graphs on underlying subgraphs (regular subgraphs, irregular subgraphs, cycles, trees) of a simple graph. We have presented regular type 2 soft graphs, irregular type 2 soft graphs, and type 2 soft trees. Moreover, we have introduced type 2 soft cycles, type 2 soft cut-nodes, and type 2 soft bridges. Finally, we have presented some operations on type 2 soft trees by presenting several examples to demonstrate these new concepts. In future work, we will extend our work in following areas of research:

(i) Applications of type 2 soft graphs in computer networks and social networks

(ii) Fuzzy type 2 soft graphs and their applications.

\section{Data Availability}

No data were used to support this study.

\section{Conflicts of Interest}

The authors declare that they have no conflicts of interest.

\section{Acknowledgments}

This paper is supported by the High Level Construction Fund of Guangzhou University China. Also this work was supported by the Natural Science Foundation of Guangdong Province (2016A030313552), the Guangdong Provincial Government to Guangdong International Student Scholarship (yuejiao [2014] 187), and Guangzhou Vocational College of Science and Technology (no. 2016TD03).

\section{References}

[1] J. A. Bondy and U. S. R. Murty, Graph Theory with Application, Macmillan Press, New York, NY, USA, 1976.

[2] A.E. Brouwer, A. M. Cohen, and A. Neumaier, Distance-Regular Graphs, vol. 18, Springer, New York, NY, USA, 1989.

[3] W. T. Tutte, Graph theory as I have known it, Oxford University Press, Oxford, England, 1998.

[4] D. Molodtsov, "Soft set theory-first results," Computers \& Mathematics with Applications, vol. 37, no. 4-5, pp. 19-31, 1999.

[5] P. K. Maji, R. Biswas, and A. R. Roy, "Soft set theory," Computers \& Mathematics with Applications, vol. 45, no. 4-5, pp. 555-562, 2003.

[6] M. I. Ali, F. Feng, X. Liu, W. K. Min, and M. Shabir, "On some new operations in soft set theory," Computers \& Mathematics with Applications, vol. 57, no. 9, pp. 1547-1553, 2009.

[7] M. I. Ali, M. Shabir, and F. Feng, "Representation of graphs based on neighborhoods and soft sets," International Journal of Machine Learning and Cybernetics, 2016.

[8] M. Akram and S. Nawaz, "Operations on soft graphs," Fuzzy Information and Engineering, vol. 7, no. 4, pp. 423-449, 2015.

[9] M. Akram and S. Nawaz, "Certain types of soft graphs," "Politehnica" University of Bucharest. Scientific Bulletin. Series A. Applied Mathematics and Physics, vol. 78, no. 4, pp. 67-82, 2016.

[10] M. Akram and F. Zafar, "On Soft Trees, Buletinul Academiei de Stiinte a Republicii Moldova," Matematika, vol. 78, pp. 82-95, 2015.

[11] R. Chatterjeea, P. Majumdar, and S. K. Samanta, "Type-2 soft sets," Journal of Intelligent \& Fuzzy Systems, vol. 29, p. 885, 2015.

[12] Y. Yang and Y. Wang, "Commentary on "Type-2 soft sets", Journal of Intelligent and Fuzzy Systems, vol. 29, pp. 885-898, 2015. 
[13] K. Hayat, M. I. Ali, B. Cao, and F. Karaaslan, "New results on type-2 soft sets," Hacettepe Journal of Mathematics and Statistics, vol. 5 , no. $46,2017$.

[14] K. Hayat, M. I. Ali, B.-Y. Cao, and X.-P. Yang, "A New Type-2 Soft Set: Type-2 Soft Graphs and Their Applications," Advances in Fuzzy Systems-Applications and Theory, vol. 2017, Article ID 6162753, 17 pages, 2017. 


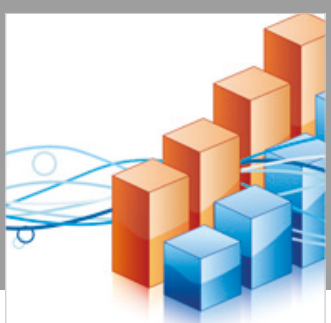

Advances in

Operations Research

\section{-n-m}
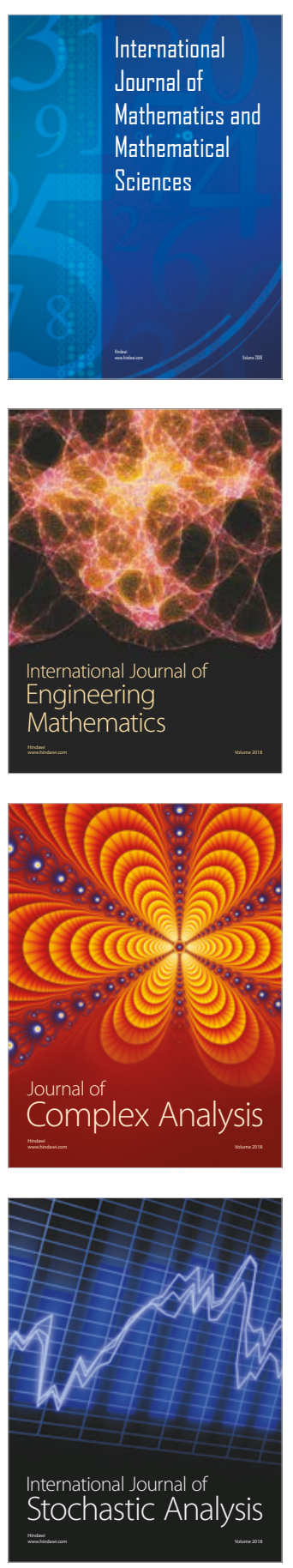
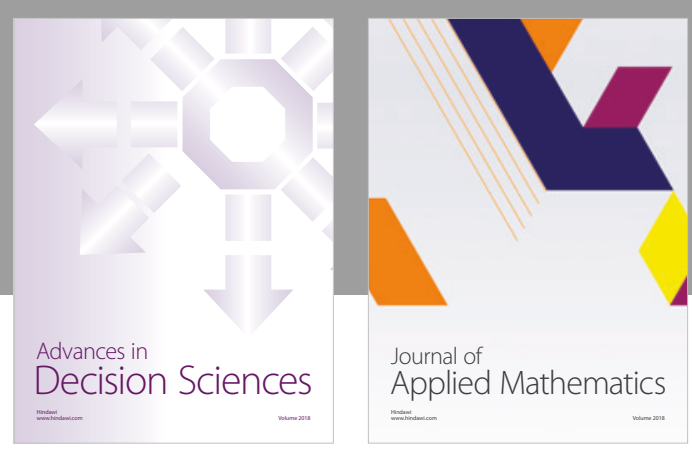

Journal of

Applied Mathematics
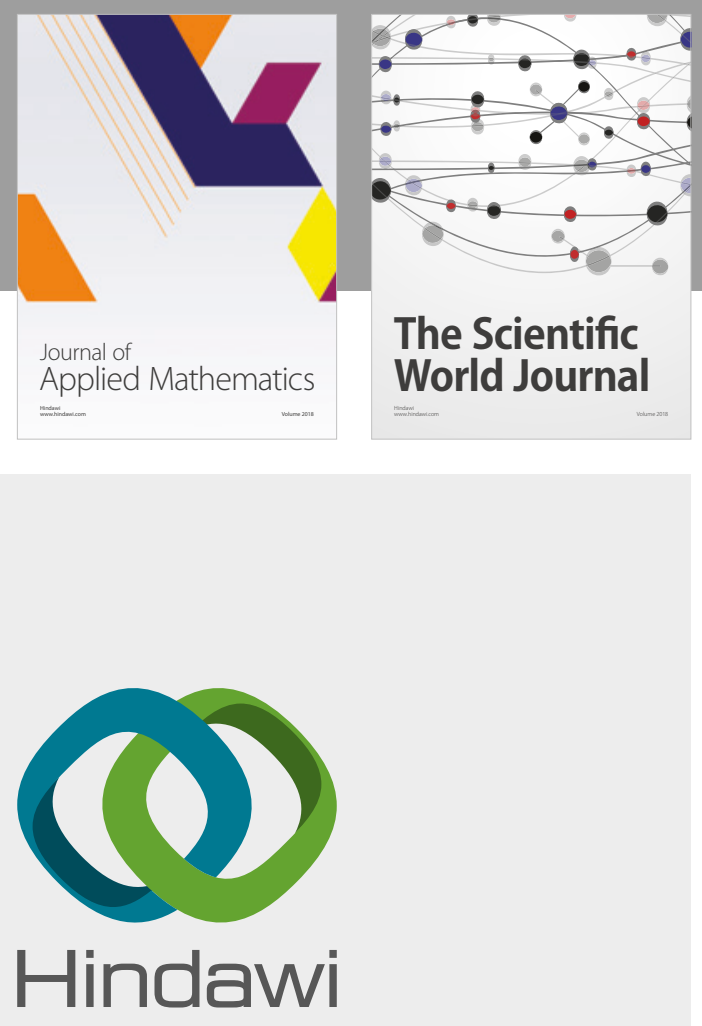

Submit your manuscripts at

www.hindawi.com

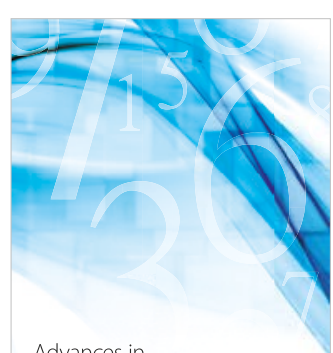

Advances in
Numerical Analysis
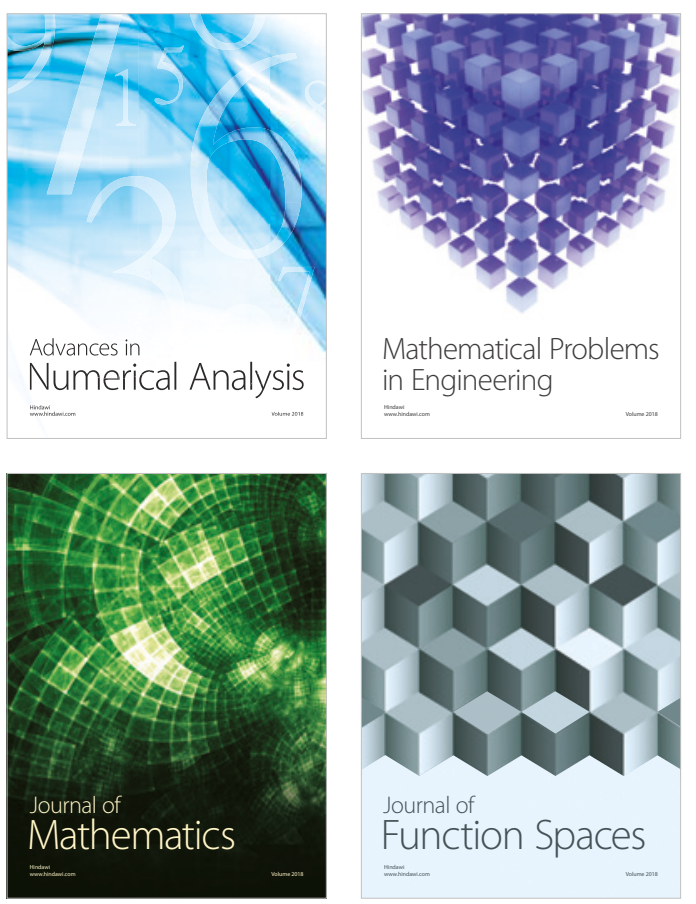

Mathematical Problems in Engineering

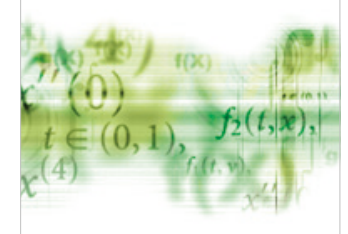

International Journal of

Differential Equations

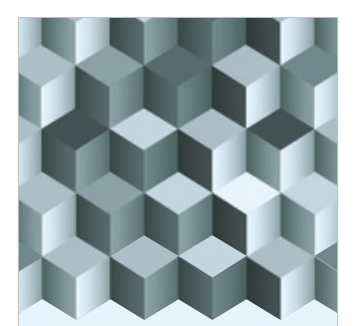

Journal of

Function Spaces

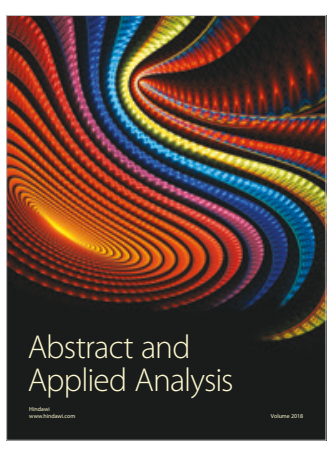

The Scientific

World Journal

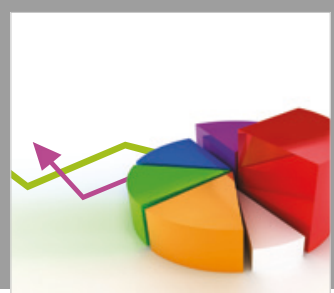

Journal of

Probability and Statistics
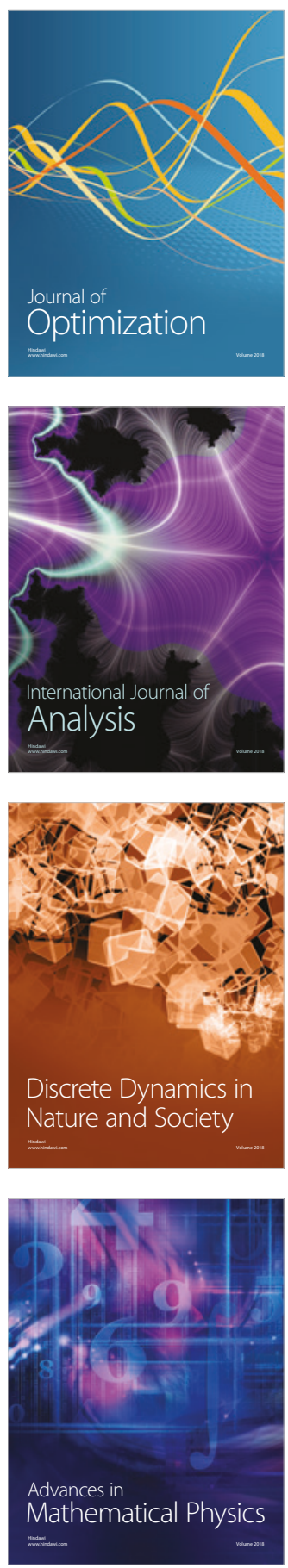